\title{
Balanced and Radiating Wave Responses to Diurnal Heating in Tropical Cyclone-Like Vortices Using a Linear Nonhydrostatic Model
}

\author{
REBECCA C. Evans AND DAVID S. NOLAN \\ Department of Atmospheric Sciences, Rosenstiel School of Marine and Atmospheric Science, \\ University of Miami, Miami, Florida
}

(Manuscript received 14 December 2018, in final form 30 May 2019)

\begin{abstract}
The diurnal cycle (DC) in the cirrus canopy of tropical cyclones (TCs) is a well-documented phenomenon. While early studies linked the DC in the area of the cirrus canopy to a DC in the strength of eyewall convection, later studies considered it a direct response to the DC of radiation in the cirrus canopy. In this study, an idealized linear model is used to examine the extent to which linear dynamics can capture the DC in TCs, in particular the transition between balanced and radiating responses to diurnal heating. The model heat forcing is physically motivated by the diabatic heating output from a realistic simulation, which illustrates the presence of a DC in moist convective heating and radiative heating in the eyewall, and a DC in radiative heating in the cirrus canopy. This study finds that the DCs of heating in the eyewall yield a response that is restricted to inside the RMW by the high inertial stability in the inner core. The DC of radiative heating in the cirrus canopy yields a response throughout the entire cyclone. Lower-frequency responses, of diurnal and semidiurnal frequency, are balanced throughout much of the cyclone. High-frequency waves with periods under $8 \mathrm{~h}$, created at sunrise and sunset, can radiate outward and downward. These results indicate that diurnal responses are balanced in the majority of a TC and originate in the cirrus canopy, instead of the eyewall. The DC in cirrus canopy vertical motion also appears to originate in the cirrus canopy.
\end{abstract}

\section{Introduction}

It has long been known that tropical cyclones (TCs) vary on the diurnal time scale, both in the strength of deep convection and in the area and height of the cirrus canopy, because of the diurnal cycle (DC) of solar radiation (Gray and Jacobson 1977; Browner et al. 1977; Muramatsu 1983; Kossin 2002). Observations of rainfall in tropical Pacific weather systems showed that the intensity of convection oscillates throughout the day, maximizing in the morning, as a result of how the differential radiative cooling between a weather system and the surrounding cloud-free region varies throughout the day (Gray and Jacobson 1977). Browner et al. (1977) and Muramatsu (1983) invoked this argument to explain the diurnal oscillation in the area of the cirrus canopy of TCs. Browner et al. (1977) noted from satellite images of Atlantic tropical storms that the daily maximum area of the cirrus canopy was 1.65 times the daily minimum area, and that the oscillation was

Corresponding author: Rebecca C. Evans, rebecca.evans@ rsmas.miami.edu weaker for stronger storms. They inferred that the cause of the areal oscillation is the DC of eyewall convection. Muramatsu (1983) elaborated on this inference using satellite observations of Pacific typhoons, arguing that the early morning convective maximum in the eyewall and spiral bands results in greater outflow, thus spreading the anvil to greater radii from the storm center, increasing the cirrus canopy area.

While these early works argued that the diurnal oscillation of the cirrus canopy area was physically linked to eyewall deep convection, later studies concluded it was a result of net radiative heating and cooling of the cirrus canopy itself (Kossin 2002; Dunion et al. 2014). In this mechanism, which was first proposed by Randall et al. (1991), daytime heating of cloud tops acts to stabilize the air column through decreasing the local lapse rate, whereas longwave radiative cooling at night increases the local lapse rate, thereby enhancing convection. Kossin (2002) argued that since the diurnal oscillation of the area of the cirrus canopy had negligible amplitude in the inner-core convective region, the oscillation in the cirrus canopy could not be mechanistically linked to eyewall convection, and is directly 
influenced by radiation through the lapse rate mechanism. Dunion et al. (2014) illustrated that for all major North Atlantic hurricanes from 2001 to 2014, a pulse of decreased infrared brightness temperature forms in the inner core of the cirrus canopy around the time of local sunset, and propagates radially outward overnight. This pulse was attributed to the lapse rate mechanism, but they also considered alternative causes for the pulse, such as the Gray and Jacobson (1977) differential cooling mechanism, or radiative cooling modifying potential vorticity of the cirrus canopy and thus the strength of outflow (Mecikalski and Tripoli 1998).

The DC has been shown to be important for TC genesis and structure. Melhauser and Zhang (2014) explored the impact of the DC of solar radiation on the pregenesis environment of Hurricane Karl (2010), finding that the night phase was critical for genesis by increasing the relative humidity and promoting convection (through the differential cooling mechanism). Similarly, Nicholls (2015) found that this mechanism accelerated the rate of cyclogenesis and enhanced nighttime convection. Tang and Zhang (2016) and Tang et al. (2017, 2019) conducted simulations of Hurricane Edouard (2014) under conditions including constant solar radiation (perpetual day), a realistic DC of insolation, and no solar radiation (perpetual night) in order to study the impact of the DC on genesis, secondary eyewall formation, and eyewall contraction during rapid intensification. In all three studies, the authors found that convection is enhanced at night because of the increase in lapse rate. Tang et al. (2019) illustrated that enhanced nighttime convection increased the vertical advection of tangential wind tendency, allowing for eyewall contraction. Furthermore, in the perpetual night case, more of the convective bursts were found inside the radius of maximum wind (RMW), which is more favorable for intensification.

Ruppert and Hohenegger (2018) and Ruppert and O'Neill (2019) studied the diurnal cycle of convection in nonrotating and TC frameworks, respectively. Ruppert and Hohenegger (2018) found that the lapse rate mechanism dictates the amplitude of the DC of precipitation, whereas the differential cooling mechanism sets the phase of the DC by delaying the peak of rainfall until the early morning. Both studies found that upper-level cooling at night causes a peak in deep convection in the lower to midtroposphere, while daytime upper-level heating induces upper-level ascent, thereby lifting the anvil cloud height. In the TC framework of Ruppert and O'Neill (2019), this daytime lifting occurs in the inner core, and propagates outward as a gravity wave, which they suggest could explain the diurnal pulses observed by Dunion et al. (2014).

Other studies on the diurnal cycle have focused on the transition between balanced and radiating waves in response to diurnal heating (Willoughby 2009; Navarro and Hakim 2016; Navarro et al. 2017; O'Neill et al. 2017). Willoughby (2009) used an idealized framework to evaluate secondary circulations produced in response to forcing with low periods, but not as low as the diurnal cycle. Navarro and Hakim (2016) and Navarro et al. (2017) used an idealized model with full physics, treating diurnal forcing as a sine wave, to find that diurnal forcing of the cirrus canopy results in a diurnal cycle of storm intensity. O'Neill et al. (2017) explored the transition between balanced and radiating waves with periods of $12 \mathrm{~h}$ or longer. They found that diurnal heating of the cirrus canopy creates a balanced response in the inner core, and radiating waves in the storm environment, with responses predominantly of diurnal and semidiurnal periods. Outside the inner core, the environment remained statically stable, supporting internal inertia-gravity waves (IIGWs).

This raises interesting questions about how a TC responds dynamically to diurnal heating. Observations of the DC in TCs are primarily from satellites, and high-spatiotemporal-resolution observations are difficult to obtain. Full-physics simulations, such as the hurricane nature run by Nolan et al. (2013) can reproduce the diurnal signals in TCs, but here we use a linear dynamical model to explore the extent to which linear dynamics can capture the DC in TCs. Specifically we aim to study the transition between balanced or radiating responses to diurnal heating in the cirrus canopy and eyewall of a TC.

\section{Methodology}

\section{a. 3DVPAS model description}

This study uses a linear model, the Three-Dimensional Vortex Perturbation Analysis and Simulation (3DVPAS), to replicate the diurnal cycles of diabatic heating from a realistic simulation of a TC and determine the dynamical responses to that heating. The 3DVPAS computes the dynamical responses to free or forced perturbations in a balanced axisymmetric vortex. The model was first derived in Nolan and Montgomery (2002) and Nolan and Grasso (2003), and later revised in Hodyss and Nolan (2007). This study uses potential temperature tendencies as forcing in 3DVPAS to imitate diabatic heating in the cirrus canopy and eyewall of a TC.

The domain used for all 3DVPAS simulations is cylindrical, extending to a radius of $800 \mathrm{~km}$ and a vertical height of $20 \mathrm{~km}$. The horizontal grid spacing is $5 \mathrm{~km}$, and 

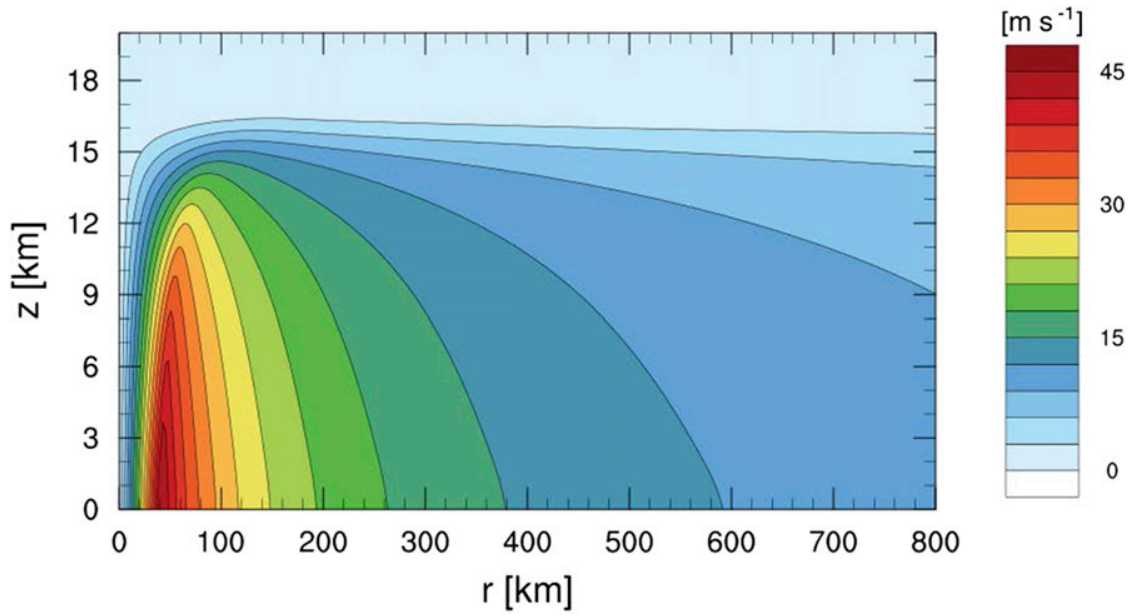

FIG. 1. The azimuthal velocity $\left(\mathrm{m} \mathrm{s}^{-1}\right)$ for the basic-state vortex with an outward-sloping RMW.

vertical grid spacing is $2 / 3 \mathrm{~km}$. The physical quantities are represented on an Arakawa $\mathrm{C}$ grid. Time stepping uses the Runge-Kutta fourth-order method. All the boundaries of the domain are closed and stress-free.

The upper and outer boundaries contain a Gaussian damping region to prevent the reflection of waves. Using trial and error, a fourth-order Gaussian was found to be the most effective for the upper boundary and a second-order Gaussian for the outer boundary. The Gaussian half width is $4 \mathrm{~km}$ for the damping zone at the upper boundary and $40 \mathrm{~km}$ for that at the outer boundary. The maximum damping rate is $1 / 1400 \mathrm{~s}^{-1}$ for the outer boundary and $1 / 60 \mathrm{~s}^{-1}$ for the upper boundary. While the damping used here prevents the reflection of waves, the damping does not perfectly absorb the waves. This results in some accumulation of wave amplitude at the edge of the domain, but no wave reflection.

The basic-state vortex for the simulations performed here is a modified Rankine vortex assuming slantwise moist neutral conditions in gradient wind balance (Emanuel 1986). The RMW at the surface is $40 \mathrm{~km}$, and the maximum tangential velocity is $45 \mathrm{~m} \mathrm{~s}^{-1}$. The velocity profile is constructed such that there are no negative tangential winds, and that the bottom $0.5 \mathrm{~km}$ is completely barotropic. The basic-state vortex is shown in Fig. 1. The method for computing the slantwise-neutral state is described in Moon and Nolan (2010). The radial and vertical flow are zero.

The axisymmetric potential temperature field $\bar{\theta}$ is constructed using a reference temperature profilethe Jordan (1958) mean tropical sounding-and the mean hydrostatic pressure field, where surface pressure is $1015 \mathrm{hPa}$. The environmental stratification is based on this potential temperature profile, where
$N^{2}=(g / \bar{\theta})(\partial \bar{\theta} / \partial z)$. The dynamic viscosity used in the simulations shown here is $40 \mathrm{~m}^{2} \mathrm{~s}^{-1}$.

The 3DVPAS is based on the linearized vortexanelastic equations on an $f$ plane as derived in Hodyss and Nolan (2007) and Nolan et al. (2007). The governing equations in 3DVPAS, shown in Eq. (1), are modified from Eqs. (3.14) in Hodyss and Nolan (2007) to remove the derivatives in the azimuthal direction since the simulations performed here are axisymmetric:

$$
\begin{aligned}
\frac{\partial u^{\prime}}{\partial t}-\left(\frac{2 \bar{v}}{r}+f\right) v^{\prime} & =-\frac{\partial}{\partial r}\left(\frac{p^{\prime}}{\bar{\rho}}\right)+F_{u}, \\
\frac{\partial v^{\prime}}{\partial t}+w^{\prime} \frac{\partial \bar{v}}{\partial z}+\left(\frac{1}{r} \frac{\partial r \bar{v}}{\partial r}+f\right) u^{\prime} & =F_{v} \\
\frac{\partial w^{\prime}}{\partial t} & =-\frac{\partial}{\partial z}\left(\frac{p^{\prime}}{\bar{\rho}}\right)+\frac{\theta^{\prime}}{\bar{\theta}}+F_{w}, \\
\frac{1}{r} \frac{\partial}{\partial r}\left(r \bar{\rho} u^{\prime}\right)+\frac{\partial}{\partial z}\left(\bar{\rho} w^{\prime}\right) & =0 \\
\frac{\partial \theta^{\prime}}{\partial t}+u^{\prime} \frac{\partial \bar{\theta}}{\partial r}+w^{\prime} \frac{\partial \bar{\theta}}{\partial z} & =F_{\theta}+\dot{Q}_{\theta} .
\end{aligned}
$$

Equations (1a)-(1c) are the momentum equations, Eq. (1d) is the anelastic mass continuity equation, and Eq. (1e) is the temperature equation. The parameters $u, v$, and $w$ are the radial, tangential, and vertical winds, respectively; $r$ and $z$ are the radial and vertical coordinates; $\rho, p$, and $\theta$ are the density, pressure, and potential temperature, respectively; and $f$ is the Coriolis parameter set to the equivalent of $20^{\circ} \mathrm{N}$. The $F$ terms denote the diffusion terms for the respective variable. Overbars denote the axisymmetric basicstate fields, which are functions of $r$ and $z$ only, and 
are not permitted to change in time, as there is no interaction of perturbations with the axisymmetric flow. Perturbations are induced in 3DVPAS using a buoyancy source in the form of a potential temperature tendency $\dot{Q}_{\theta}(r, z, t)$.

\section{$b$. The hurricane nature run as inspiration for the 3DVPAS forcing}

The spatial and temporal forcing used in the 3DVPAS simulations was motivated by the diabatic heating output from the hurricane nature run (HNR) (Nolan et al. 2013). The HNR is a high-resolution, full-physics simulation of a realistic, mature TC. It was chosen as the physical motivation for the 3DVPAS heat forcing on the basis of its high resolution and accurate representation of the interaction of clouds and radiative heating. We use the output from 0000 UTC 3 August to 2330 UTC 9 August, when the HNR TC was at hurricane intensity. The HNR TC underwent rapid intensification on 3 August followed by an eyewall replacement on 4 August, reaching peak intensity at $60 \mathrm{~m} \mathrm{~s}^{-1}$ on 5 August, then slowly weakening to around $45 \mathrm{~m} \mathrm{~s}^{-1}$ by 0000 UTC 10 August. To confirm that the rapid intensification and weakening did not project on to the diurnal frequency, we also considered the period when the TC was in approximately steady state, finding that the results were very similar.

This study uses the azimuthally averaged fields of radiative heating (shortwave and longwave) and moist convective heating to determine the spatial structure of heating in a realistic TC and how the heating evolves throughout the course of a day. These were then idealized, as described later, such that they could be used as heat forcing in 3DVPAS.

\section{c. Forcing in $r-z$ space}

Using the output of azimuthally averaged radiative heating from the HNR, a DC in radiative heating was found to be present in the cirrus canopy and eyewall of a TC (Fig. 2a). Figure 2 was created by generating a time series of radiative or moist convective heating for each $(r, z)$ grid point of the azimuthally averaged fields, computing the power spectrum for that time series, and finding the amplitude corresponding to exactly 1 day $^{-1}$ (the DC). The warmer colors show where the amplitude of the DC is greatest, illustrating that the DC of radiative heating is strongest in the dense clouds of the cirrus canopy, with an amplitude of around $0.4 \mathrm{~K} \mathrm{~h}^{-1}$, and is much weaker in the eyewall. This is because the high opacity of the cirrus clouds results in strong absorption of solar radiation, and therefore a large DC. The DC of radiative heating is weaker in the eyewall, with an amplitude of $0.12 \mathrm{~K} \mathrm{~h}^{-1}$, since the clouds overlying the eyewall absorb much of the incoming radiation, preventing solar heating of the clouds below. While there is a very weak DC in longwave (LW) heating above and below the cirrus canopy (not shown), the maxima in amplitude are less than $0.1 \mathrm{~K} \mathrm{~h}^{-1}$ and elsewhere in the domain the LW heating is essentially constant throughout the day. As such, the DC in total radiative heating in Fig. 2a is dominated by the DC in shortwave (SW) heating.

Figure 2a illustrates the average position of the cirrus canopy. The maximum radiative heating of the cirrus canopy is at a height of around $13 \mathrm{~km}$ around the RMW. The cirrus canopy extends a few tens of kilometers inward of the RMW, with the eye remaining clear, and outward to a radius of around $300 \mathrm{~km}$. The maximum vertical extent of the cirrus canopy stands at $16 \mathrm{~km}$ near the eyewall and descends to $12 \mathrm{~km}$ at $300-\mathrm{km}$ radius. The cirrus canopy also thins with increasing radius from the RMW. The maximum heating is displaced around $5 \mathrm{~km}$ outside the RMW. The average position of the eyewall is illustrated better by the DC of moist convective heating in Fig. 2b, which shows that the DC of moist convective heating is strongest in the eyewall of the TC, which is displaced $10-20 \mathrm{~km}$ inward of the RMW. Additionally there is a sizeable oscillation in the outer rainbands that will not be discussed further here but could be illustrative of a DC in rainband activity, as discussed in Dunion et al. (2019). The amplitude of the DC of moist convective heating in the eyewall is an order of magnitude greater than that of radiative heating. While the underlying cause of the DC of moist convective heating is unclear, it most likely induces a specific dynamical response.

Using the above as inspiration for the spatial pattern of heating in TCs, idealized potential temperature perturbations were constructed to use as heat forcing in 3DVPAS. These idealized forcings are shown in Fig. 3. As in the HNR, the idealized cirrus canopy in the 3DVPAS simulations is centered at $13 \mathrm{~km}$, and extends to a radius of $300 \mathrm{~km}$, thinning with increasing radius from the RMW. The idealized eyewall extends from a height of around $2-11 \mathrm{~km}$, and the heating maximum is displaced around $10 \mathrm{~km}$ inward of the RMW, sloping outward with height congruently with the slope of the RMW. The maximum heating, indicated by deepest red color, is here normalized to $1 \mathrm{~K} \mathrm{~h}^{-1}$ to show the spatial pattern of prescribed heating. However, the value of the maximum heating is designed to evolve with time, according to the temporal forcing for the respective simulation, as described in section 2d. Mathematical formulas for these potential temperature perturbations can be found in the appendix. 
(a) Amplitude of the DC in the HNR: Radiative heating (SW+LW)
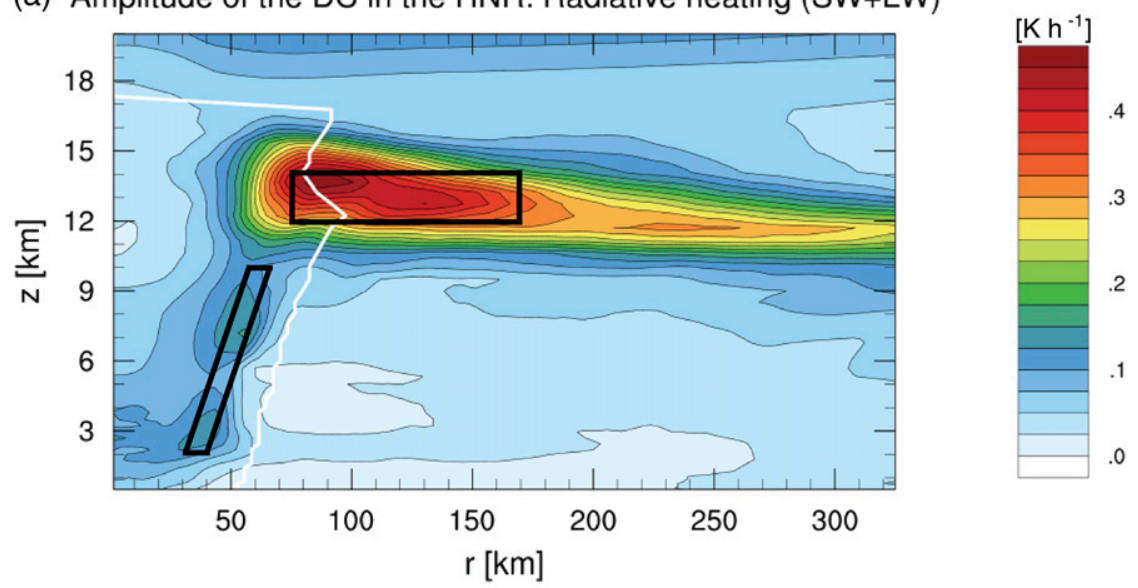

(b) Amplitude of the DC in the HNR: Moist convective heating
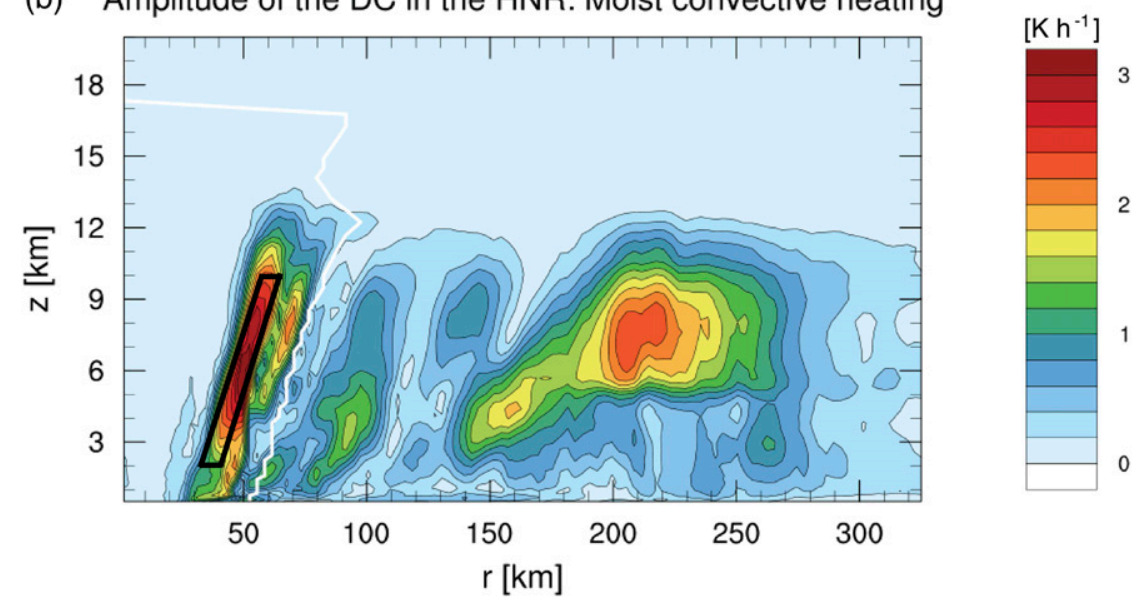

FIG. 2. Two $r-z$ maps of the amplitude of the DC in heating rate for (a) radiative heating and (b) moist convective heating in the Hurricane Nature Run (HNR). Red colors denote where the amplitude of DC is the largest. The white line is the mean position of the RMW. The black boxes denote the regions over which area averages were taken.

\section{d. Forcing in time}

The temporal forcing used in 3DVPAS was inspired by the diurnal cycles for moist convective heating and radiative heating in the cirrus canopy and eyewall of the TC in the HNR. The diurnal cycles for each of these are shown in the left panels of Fig. 4.

These diurnal cycles were created by taking an area average in $(r, z)$ space over the cirrus canopy and eyewall. For the eyewall, the area average was taken over a fixed box centered at the heating maximum in Fig. 2a that extended from 2- to $10-\mathrm{km}$ height, and was $10 \mathrm{~km}$ wide, sloping outward parallel to the RMW. To test whether artificial diurnal variability was being captured by the heating maximum simply moving in and out of the box, we also conducted area averages using an eyewall-following technique, whereby the area average was taken everywhere inside $100-\mathrm{km}$ radius that moist convective heating exceeded 10,15 , or $20 \mathrm{~K} \mathrm{~h}^{-1}$. This had little effect on the result, except for the amplitude of the DC. For the purposes of this study, the amplitudes are not important, since we are using a linear model. The area average for the cirrus canopy was taken over a box, from a radius of $75-170 \mathrm{~km}$ and height of $12-14 \mathrm{~km}$.

The $r$ and $z$ limits of the boxes in which the area average was performed were chosen to capture as much diurnal variability as possible. For the cirrus canopy, the box was chosen to be vertically centered between the daytime maximum heating, and nighttime maximum cooling, since the center of the cirrus canopy moved up and down throughout any given day. By choosing to perform an area average over the described box, the diurnal variability was captured even though 
(a) $\dot{Q}_{\theta}$ : Idealized 3DVPAS Cirrus Canopy

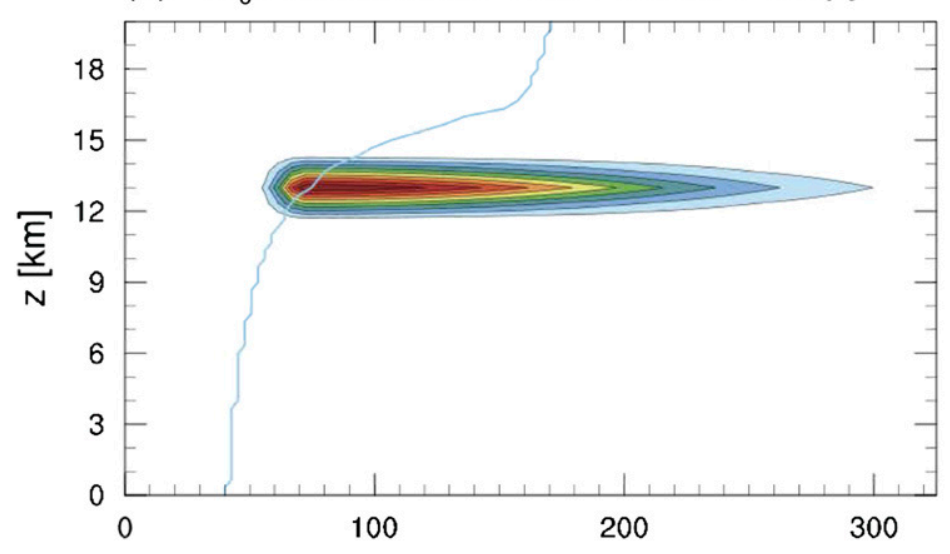

(b) $\quad \dot{Q}_{\theta}$ : Idealized 3DVPAS Eyewall

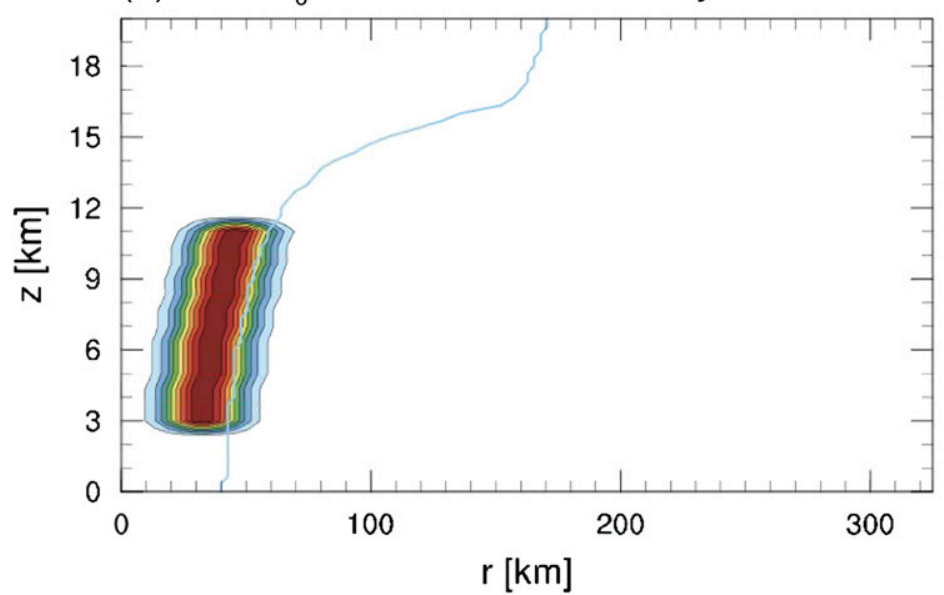

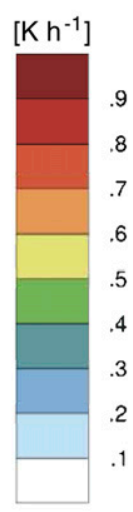

$\left[\mathrm{Kh}^{-1}\right]$

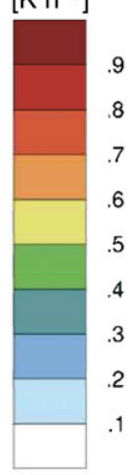

FIG. 3. Idealized potential temperature tendencies used as heat forcing in 3DVPAS aiming to replicate (a) the cirrus canopy and (b) the eyewall. The maximum heating (deepest red color) is here normalized to $1 \mathrm{~K} \mathrm{~h}^{-1}$, as described in the text. The blue line is the RMW for the 3DVPAS basic-state vortex. Although the domain extends to $800-\mathrm{km}$ radius, only $\dot{Q}_{\theta}$ has been shown to $325 \mathrm{~km}$ to facilitate comparison with the HNR heating in Fig. 2.

the daytime maximum and nighttime minimum were in different vertical locations. In the eyewall, the diurnal variability was captured even though the exact position of the maximum heating within the eyewall varied with time. Of course, the amplitudes of the heating are lower than the actual maximum heating for both the cirrus canopy and the eyewall, since the area average includes regions in which heating is weaker. After performing the area average, the resultant time series over the entire HNR for moist convective heating in the eyewall and radiative heating in the eyewall and cirrus canopy were low-pass filtered to remove frequencies less than $6 \mathrm{~h}$; then a daily composite was taken of this filtered data. The low-pass-filtered daily composites are shown in the left panels of Fig. 4. This illustrates that the DC for moist convective heating in the eyewall is sinusoidal in shape, with a maximum approximately $3 \mathrm{~h}$ before local sunset, and minimum $3 \mathrm{~h}$ before sunrise. While the exact timing is not important here, since this study uses a linear model, the peak in eyewall convection occurs at a different time than the observed early morning peak in TC rainfall (Bowman and Fowler 2015). The peak of the integrated rainfall throughout the entire HNR TC is however consistent with observations, as the rainband rainfall, which appears to dominate the diurnal rainfall signal, peaks in the early morning (not shown). For radiative heating, the DC shows uniform cooling from sunset (0000 UTC) to sunrise (0900 UTC), and heating during the day, peaking at 1500-1600 UTC. These functions are the sum of shortwave heating, which is zero at night and approximately sinusoidal in shape during the day, plus approximately constant longwave cooling. 

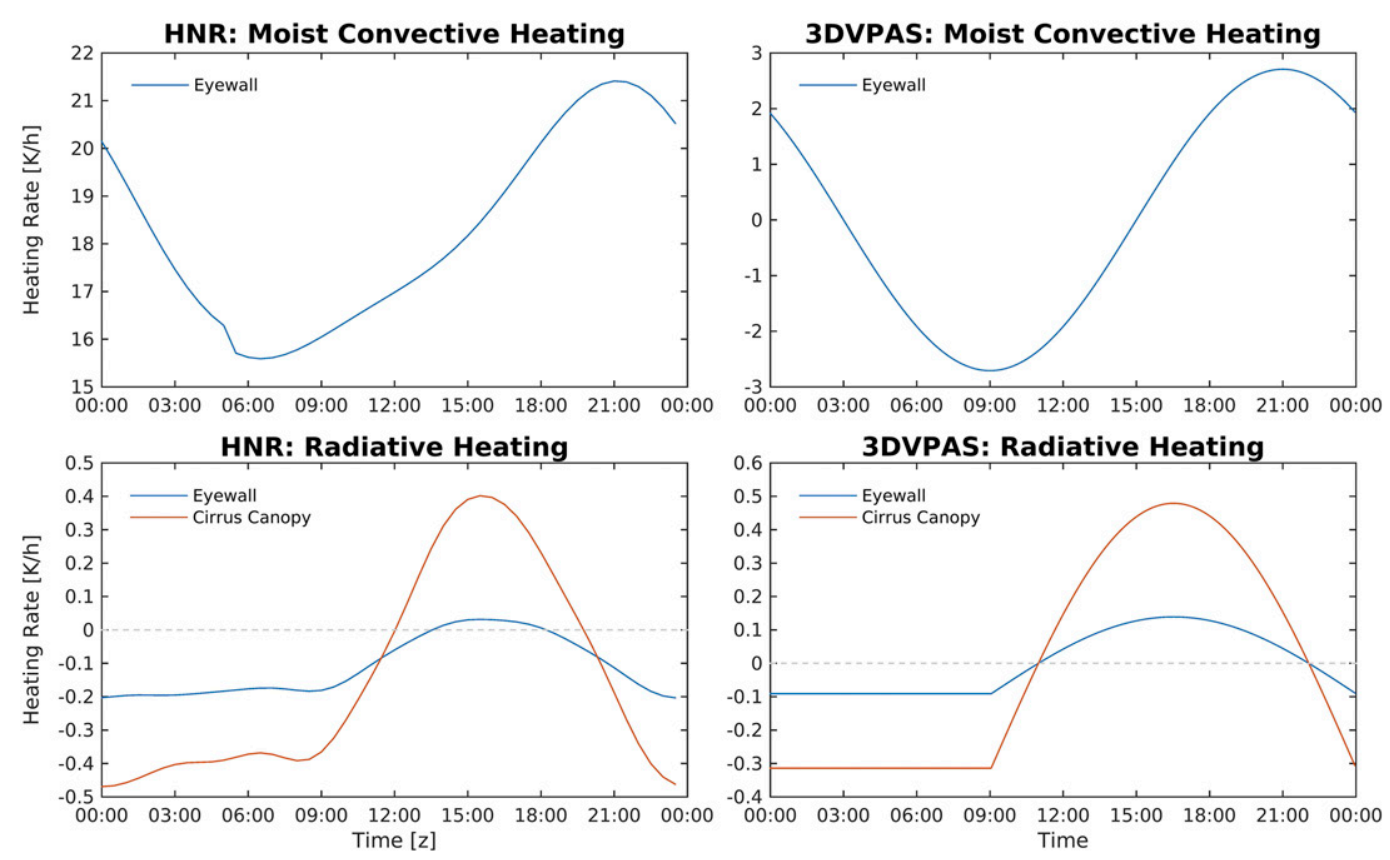

FIG. 4. The diurnal cycles of radiative and moist convective heating in (left) the HNR and (right) the idealized versions for 3DVPAS. (top) DC for moist convective heating and (bottom) radiative heating for the cirrus canopy (red) and eyewall (blue). Note the change in scale between the panels for the HNR and those for 3DVPAS, as well as the change to a mean of zero for the diurnal cycles in the 3DVPAS panels.

Note that the mean of the DC of radiative heating in the eyewall is negative, since the constant longwave emission by the clouds has a greater net effect than the heating by solar radiation during the day.

These heating rates were then idealized for use as temporal forcing functions in 3DVPAS, such that the potential temperature perturbations representing the cirrus canopy and eyewall evolved with time, imitating the realistic DC of radiative and moist convective heating. These idealized functions are shown in the right panels of Fig. 4. Note that the forcing functions for all the simulations were adjusted to have a mean of zero to prevent a secular trend in the 3DVPAS response, and to show anomalies to the mean. The idealized DCs were then applied to the appropriate idealized spatial forcing (cirrus canopy or eyewall) and repeated for four model days to determine the dynamical responses to diurnal heating in the cirrus canopy and eyewall of a TC. A mathematical representation of the idealized temporal forcing functions can be seen in the appendix.

\section{Results}

For all the simulations discussed below, the dynamical responses at specific times will be presented in the order $\theta^{\prime}$, then $w^{\prime}$, then $u^{\prime}$, and then $v^{\prime}$. This order was chosen as the prescribed heating generates buoyancy through $\theta^{\prime}$, which then causes vertical motion $w^{\prime}$. The vertical motion induces a secondary circulation, and therefore $u^{\prime}$, because of mass conservation. The $u^{\prime}$ then causes changes in tangential wind $v^{\prime}$ because of the conservation of angular momentum. Often the $w^{\prime}$ field appears to respond to a change in sign of the prescribed heating before $\theta^{\prime}$, which is a result of $w^{\prime}$ compensating for the heating through adiabatic cooling or warming before $\theta^{\prime}$ has accumulated.

\section{a. Moist convective heating in the eyewall}

For the simulation replicating moist convective heating in the eyewall, the eyewall-like potential temperature tendency from Fig. $3 \mathrm{~b}$ is forced to heat and cool according to the top-right panel of Fig. 4. The heating and cooling produces a dynamical response, shown in Figs. 5-7. The times at which snapshots were taken correspond to the maximum heating, zero heating, and maximum cooling. These snapshots were taken around halfway through the simulation, at 1.875, 2.125, and 2.375 days; however, it should be noted that each model day has the exact same forcing and response, since this is a linear model. Therefore, the specific day of the snapshots is arbitrary, and only the timing relative to maximum and minimum heating is important.

At the time of the prescribed heating maximum, as in Fig. 5 , it is evident that the actual $\theta^{\prime}$ is weakly negative in 


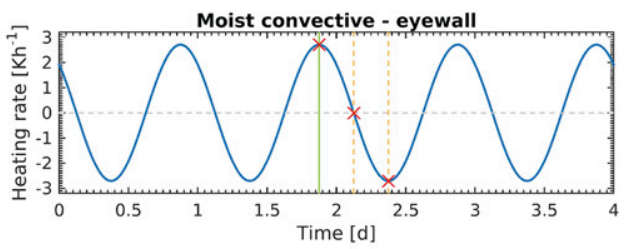

(a)

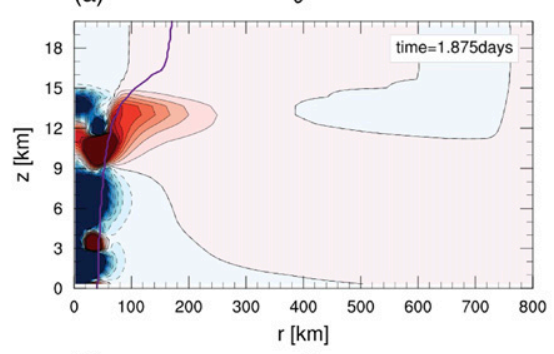

(c)

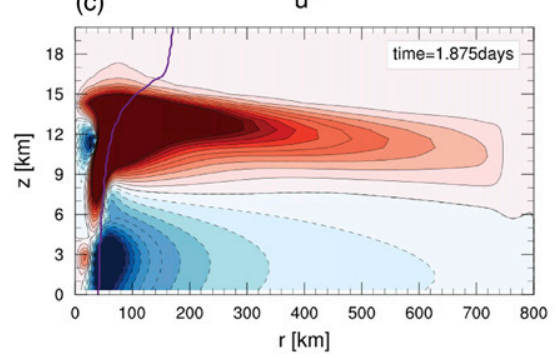

(b)

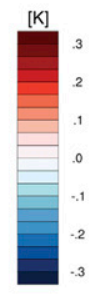

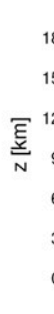

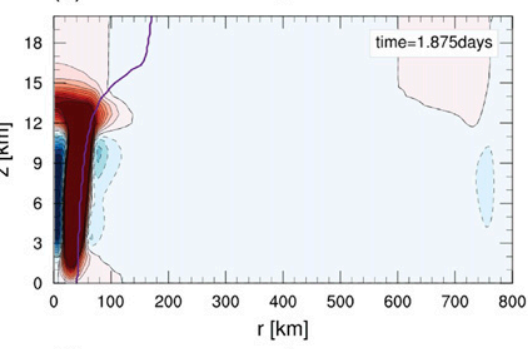

(d)

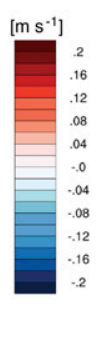

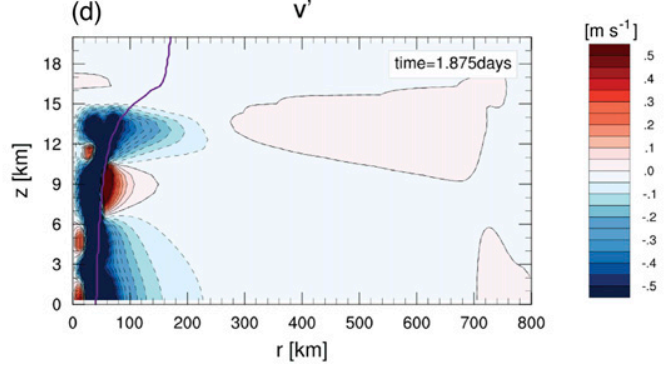

FIG. 5. Moist convective heating in the eyewall at 1.875 days, the prescribed heating maximum. $r-z$ snapshots of (a) $\theta^{\prime}(\mathrm{K}),(\mathrm{b}) w^{\prime}\left(\mathrm{m} \mathrm{s}^{-1}\right)$, (c) $u^{\prime}\left(\mathrm{m} \mathrm{s}^{-1}\right)$, and (d) $v^{\prime}\left(\mathrm{m} \mathrm{s}^{-1}\right)$. The color scale is saturated at $10 \%$ of the maximum to observe finer details of the response. (top) The temporal forcing used in the simulation. The green line denotes the time at which the snapshots in (a)-(d) were taken. The orange lines and red markers denote the times at which the snapshots in the other panels for the simulation were taken. The purple line is the RMW.

much of the eyewall, even though there has been heating for several hours. This is due to the lag between when heating is prescribed and its accumulated effect, so at this time, $\theta^{\prime}$ is in the process of transitioning between negative and positive in the eyewall. The value of $\theta^{\prime}$ becomes positive first at the top and bottom of the eyewall, filling in toward the vertical center of the eyewall. The prescribed heating induces vertical motion $w^{\prime}$ to compensate for the heating such that at the time of the prescribed heating maximum, a secondary circulation is induced throughout the entire cyclone. This change in the secondary circulation has a shorter lag behind the prescribed heating than the changes in $\theta^{\prime}$. In response to the heating, there is localized positive $w^{\prime}$, which for some time balances the heating through adiabatic cooling. The heating is instead realized elsewhere as adiabatic warming through subsidence. As heating continues, $w^{\prime}$ and adiabatic cooling can no longer fully compensate for the heating, and so $\theta^{\prime}$ begins to respond. Eyewall heating results in upward motion (positive $w^{\prime}$ ) in the eyewall, and compensating downward motion in the eye and outside the RMW to conserve mass. At this time, there is strong divergent motion in the upper levels, and convergence in the lower levels, as shown by $u^{\prime}$ in Fig. 5c. The upper-level outward flow extends to a larger radius than the lower-level inward flow. In the upper levels the density is lower than in the lower levels; thus, upper-level $u^{\prime}$ is stronger to conserve mass. The positive $u^{\prime}$ in the upper eyewall leads to negative $v^{\prime}$ because of the conservation of angular momentum. At this time $v^{\prime}$ is negative, by more than $0.5 \mathrm{~m} \mathrm{~s}^{-1}$ throughout the entire depth of the eyewall. Eyewall $v^{\prime}$ does not respond uniformly to the heating; at innermost $r, v^{\prime}$ alternates between positive and negative with increasing $z$. This pattern begins to show around $3 \mathrm{~h}$ before the peak heating, and demonstrates the transition between $v^{\prime}$ typical of cooling and $v^{\prime}$ typical of heating.

A few hours after the peak heating, as in Fig. 6, $\theta^{\prime}$ has responded to the prescribed heating, with a strong positive $\theta^{\prime}$ throughout the eyewall. Although the temporal forcing function has zero heating rate at this time, motion is nonzero, since the dynamical response of the atmosphere lags behind the change in heating. There is a weak positive $w^{\prime}$ in the uppermost eyewall at a height of 


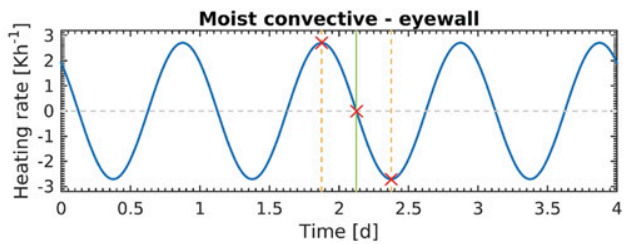

(a)

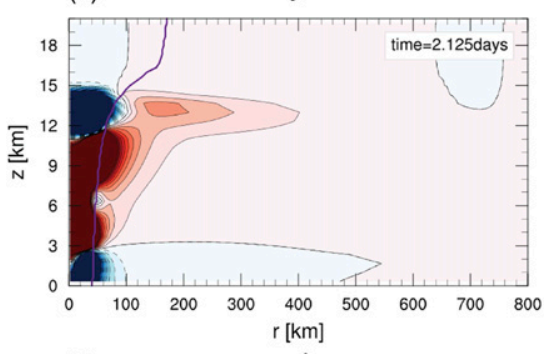

(c)

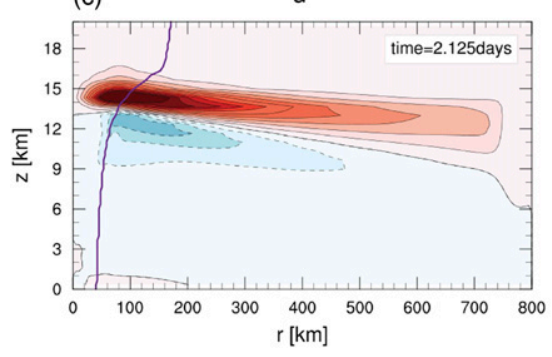

(b)
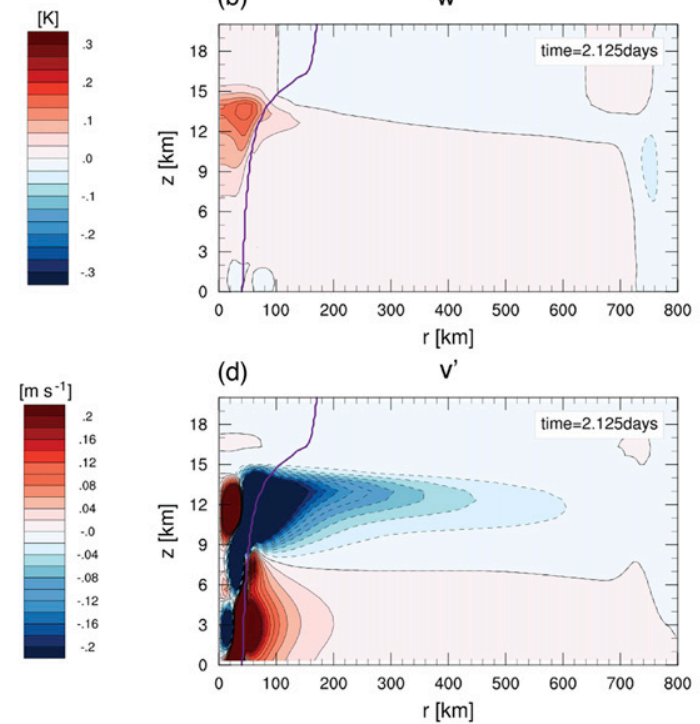

'

(d)

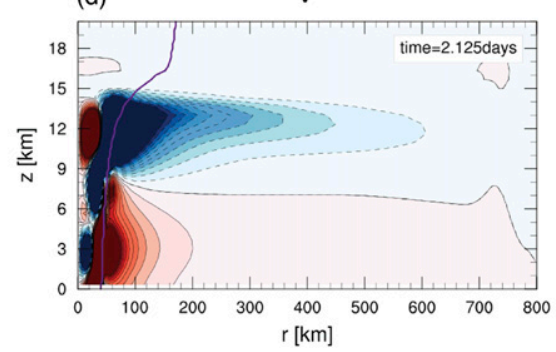

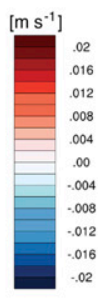

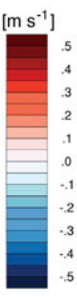

FIG. 6. As in Fig. 5, but for time $=2.125$ days, when prescribed heating is zero.

$12-15 \mathrm{~km}$. This results in positive $u^{\prime}$ above $13 \mathrm{~km}$, and a weak negative $u^{\prime}$ below. Thus, a weak high-level secondary circulation remains even at the moment when prescribed heating is zero. The circulation is confined to the upper levels of the cyclone, at $12-15 \mathrm{~km}$. The positive $u^{\prime}$ induces negative $v^{\prime}$, illustrating anomalously anticyclonic motion.

At the maximum cooling, as in Fig. 7, $\theta^{\prime}$ has not yet reached its minimum in the entire eyewall, and is in the process of transitioning from a state typical of eyewall heating to that of eyewall cooling. At this time, the secondary circulation is the opposite to that of the maximum heating, with downward motion in the eyewall, upward motion elsewhere, convergence in the upper levels, and divergence in the lower levels. It should be noted that in a realistic tropical cyclone, cooling does not necessarily result in downward motion, but in a linear dynamical model such as this, changing the sign of prescribed heating yields a change in sign of vertical motion. It also appears that the secondary circulation responds more quickly to a change in sign of prescribed heating than $\theta^{\prime}$ and $v^{\prime}$. The negative $u^{\prime}$ in the uppermost levels induces positive $v^{\prime}$ in the uppermost eyewall. In the lower levels, below $9 \mathrm{~km}$, this is the opposite. The $v^{\prime}$ response to prescribed heating only appears to take effect when the prescribed heating approaches its maximum or minimum. Therefore, much like $\theta^{\prime}, v^{\prime}$ accumulates over time.

All snapshots of $w^{\prime}$ in Figs. 5-7 illustrate that the motion produced in response to heating from moist convection in the eyewall is entirely balanced, with no radiating response. In a linear model, the spectrum of frequencies that make up the temporal forcing function should also manifest itself in the response. Since the temporal forcing function used in this simulation is a sinusoidal wave of exactly diurnal frequency, the frequency of the response is also entirely diurnal. The lack of radiating response can be understood by considering where gravity waves of varying frequencies can radiate in a weakly baroclinic tropical cyclone. This can be found using Eq. (11) from Willoughby (2009), which describes a passband of frequencies permissible for the radiation of gravity waves. The components of the equation are shown in Eq. (2) taken from Willoughby (2009), and the passband of frequencies is Eq. (11) from Willoughby (2009), shown here as Eq. (3). This equation also comprises a central focus of the studies by Navarro et al. (2017) and O'Neill et al. (2017). The inertial stability squared $I^{2}$ is equal to the absolute vorticity times the inertial parameter, $\gamma$ is defined as the ratio of horizontal acceleration to gravity, $B$ is the radial gradient of the mean vortex 

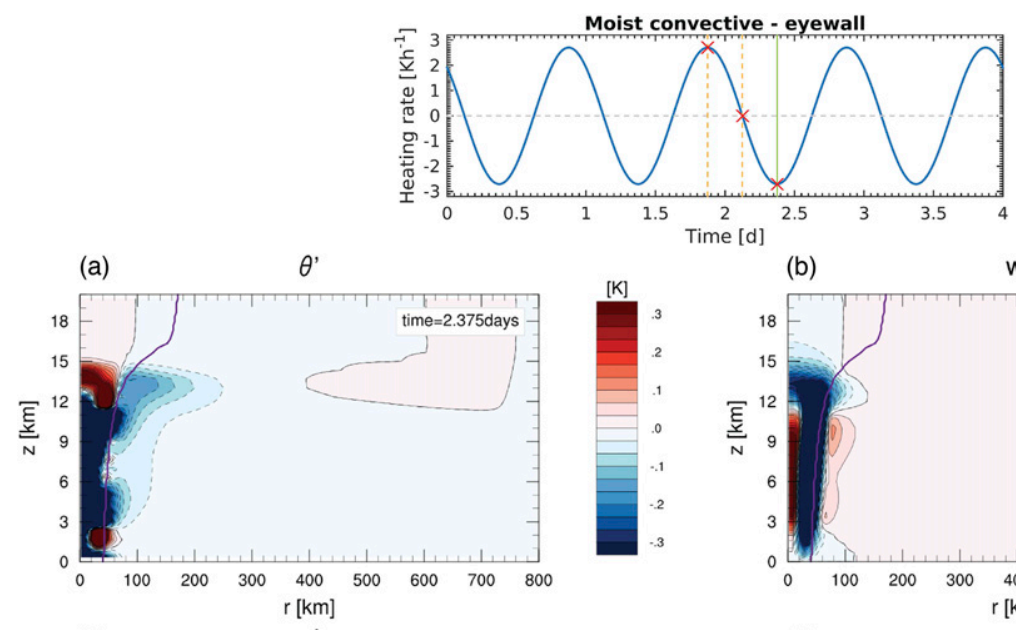

(b) $\quad w^{\prime}$
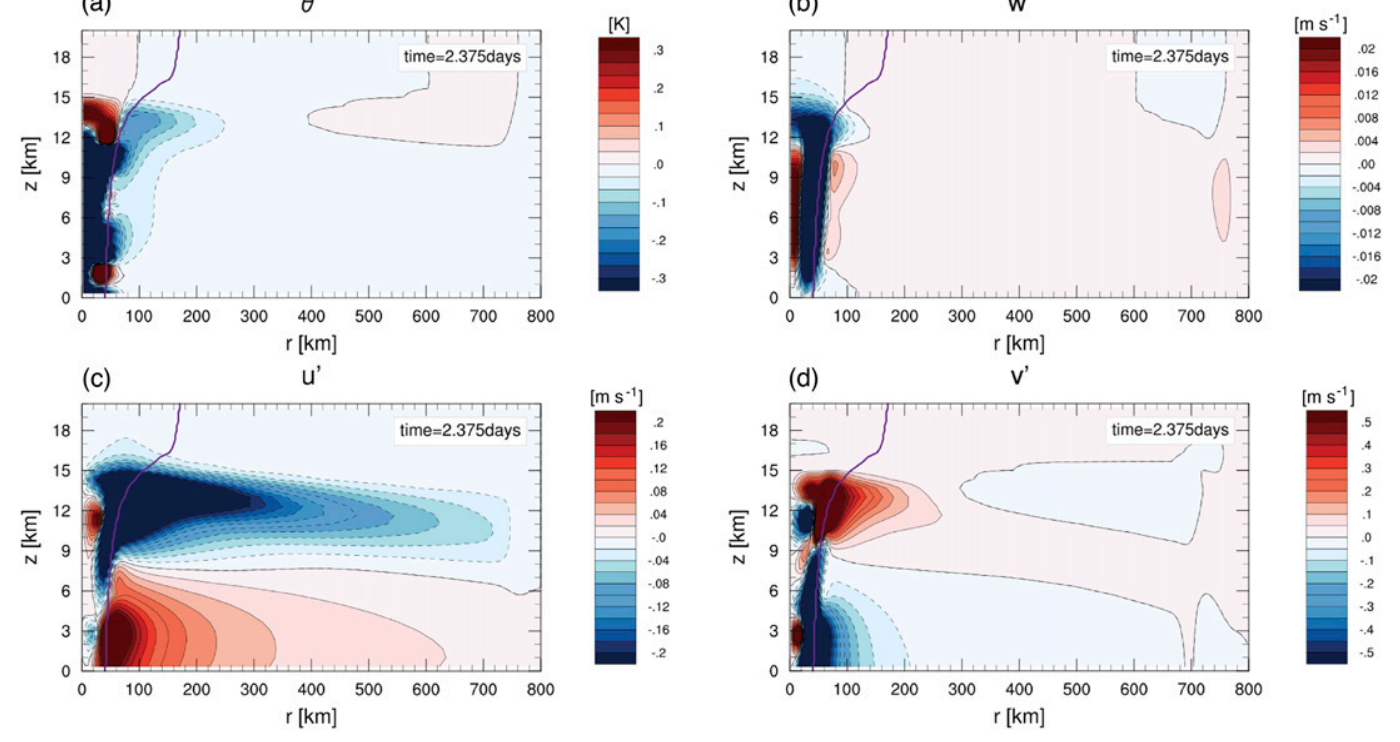

FIG. 7. As in Fig. 5, but for time $=2.375$ days, the prescribed cooling maximum.

buoyancy, and $N^{2}$ is the stratification, as defined before. Together, these yield the isobaric inertial stability $I^{\prime 2}$ :

$$
\begin{aligned}
I^{2} & =\left(\frac{\partial \bar{v}}{\partial r}+\frac{\bar{v}}{r}+f\right)\left(\frac{2 \bar{v}}{r}+f\right), \\
\gamma & =\frac{1}{g}\left(\frac{\bar{v}^{2}}{r}+f \bar{v}\right), \\
B & =\frac{\partial}{\partial r}\left[g \ln \left(\frac{\bar{\theta}}{273.16 \mathrm{~K}}\right)\right], \\
I^{2} & =I^{2}-\gamma B^{2}, \\
I^{\prime 2}-\frac{1}{2} & \frac{B^{2}}{N^{2}-I^{\prime 2}} \leq \omega^{2} \leq N^{2}+\frac{1}{2} \frac{B^{2}}{N^{2}-I^{2}} .
\end{aligned}
$$

For waves of any given frequency to be able to radiate, their frequency must be greater than the left-hand side of Eq. (3). In our basic-state vortex, the left-hand side of Eq. (3) is almost equal to $I^{2}$ as the second term is very small. Therefore, the regions in which gravity waves can radiate are dictated by the inertial stability of the basicstate vortex. For the diurnal frequency, $\omega$ is equal to $2 \pi \mathrm{d}^{-1}$, or $7.29 \times 10^{-5} \mathrm{~s}^{-1}$, as dictated by the pendulum day. At the diurnal frequency, $\omega^{2}$ exceeds $I^{2}$ in the outer regions of a vortex, where winds approach zero, and thus in these regions gravity waves of diurnal frequency can radiate. As winds approach zero, $I^{2}$ approaches $f^{2}$, which at $20^{\circ} \mathrm{N}$ is too small to prevent the radiation of diurnal waves. At higher latitudes this would not be true. At $20^{\circ} \mathrm{N}$ the response to heating is balanced for diurnal frequencies in the majority of the domain. Much of the dynamical response due to diurnal heating of the eyewall is confined to inside the RMW, where $\partial \bar{v} / \partial r$ is positive, owing to the high inertial stability in the inner core of the vortex.

\section{b. Radiative heating in the eyewall}

For the simulation replicating radiative heating in the eyewall, the eyewall-like potential temperature tendency is forced to heat and cool according to the blue line in the lower-right panel of Fig. 4. The dynamical responses are shown in Figs. 8-11. The snapshots are taken at the points in the temporal forcing corresponding to the model sunrise, between sunrise and maximum heating, maximum heating, and model sunset.

At model sunrise, as in Fig. 8a, the spatial distribution of $\theta^{\prime}$ is typical of that of eyewall cooling, with negative $\theta^{\prime}$ in the eyewall and positive $\theta^{\prime}$ above and below the eyewall. The secondary circulation, in 


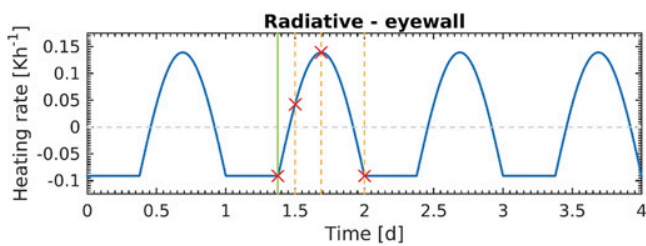

(a)

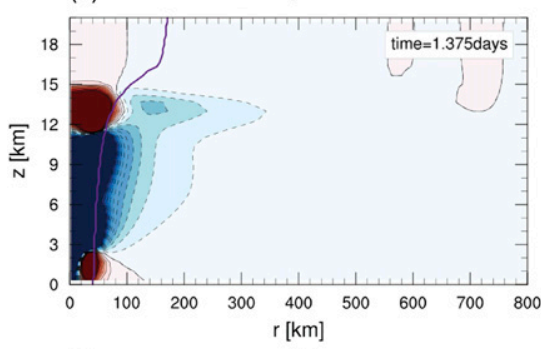

(c)

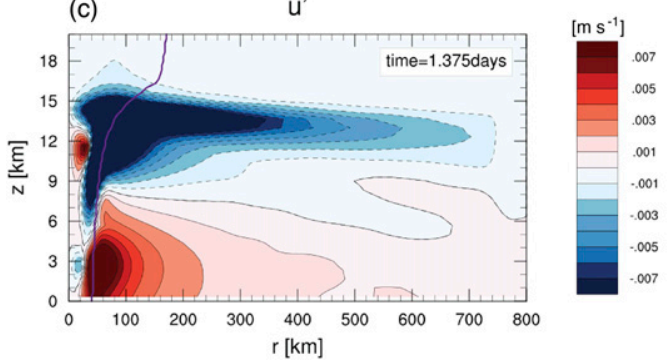

(b)

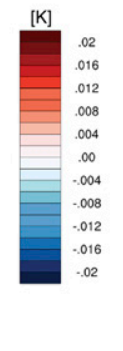

18
15
站 $^{12}$
9
6
3
0 $w^{\prime}$

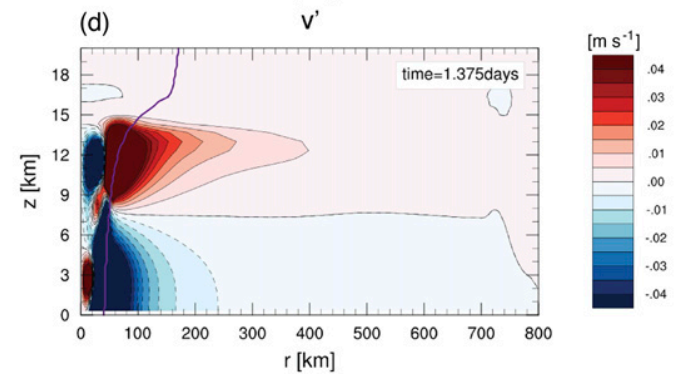

FIG. 8. Radiative heating in the eyewall at 1.375 days, the model sunrise. $r-z$ snapshots of (a) $\theta^{\prime}(\mathrm{K}),(\mathrm{b}) w^{\prime}\left(\mathrm{m} \mathrm{s}^{-1}\right)$, (c) $u^{\prime}\left(\mathrm{m} \mathrm{s}^{-1}\right)$, and (d) $v^{\prime}\left(\mathrm{m} \mathrm{s}^{-1}\right)$. The saturation of the color scale, and the line and marker colors are as in Fig. 5.

Figs. $8 \mathrm{~b}$ and $8 \mathrm{c}$, is very similar at this time to that of the maximum cooling for eyewall moist convective heating. Eyewall $w^{\prime}$ is negative, with compensating upward motion in the eye and outer cyclone. Upperlevel $u^{\prime}$ is convergent, and lower-level $u^{\prime}$ is divergent. However, in $w^{\prime}$ at heights below $6 \mathrm{~km}$ and radii from 180 to $620 \mathrm{~km}$, waves appear to be present, as shown by $w^{\prime}$ alternating between positive and negative with increasing $r$. In Fig. 8d, outside a radius of $20-40 \mathrm{~km}, v^{\prime}$ is negative in the lower levels and positive in the upper levels. This reflects the spatial pattern of $u^{\prime}$, as outward motion in the lower levels results in a negative $v^{\prime}$, and vice versa for the upper levels. Accordingly, at radii inward of $20-40 \mathrm{~km}$, between 0 - and $5-\mathrm{km}$ heights there is positive $v^{\prime}$ in the lower levels, and negative $v^{\prime}$ in the eye in the upper levels. This again reflects the spatial distribution of $u^{\prime}$ in the eye.

Between sunrise and maximum heating, the heat forcing has become positive but the spatial distribution of $\theta^{\prime}$ remains typical of that of eyewall cooling, as shown in Fig. 9a. Furthermore, there are some waves at this time, as evident by the alternating positive and negative $\theta^{\prime}$ at the top of the domain. The secondary circulation is substantially more complicated than before, as the circulation begins to respond to the change in sign of the heat forcing. There is now upward motion throughout the eyewall, as shown in Fig. 9b, with weak compensating downward motion in the eye and outside the RMW. There is still remnant negative $w^{\prime}$ in the uppermost eyewall, at a height of $13 \mathrm{~km}$, from the previous cooling, as it is located outside the heating zone, and so takes more time to respond to the prescribed heating. This induces a weak counterclockwise secondary circulation at heights above $12 \mathrm{~km}$, and a clockwise circulation below, as evident by the spatial pattern of $u^{\prime}$ in Fig. 9c. Waves are most evident in the $\theta^{\prime}$ and $w^{\prime}$ fields in the upper part of the domain, above $13 \mathrm{~km}$. At this time, as with $\theta^{\prime}$, the $v^{\prime}$ spatial distribution remains largely the same as the before, since it has not yet responded to the prescribed positive heating.

At the peak heating, as in Fig. 10, the spatial distributions of the responses for all variables are almost exactly the same as those in Fig. 5, with the exception of the waves at radii beyond $140 \mathrm{~km}$ and heights below $10 \mathrm{~km}$ in Figs. 10a and 10b. The waves appear to radiate outward and downward from the eyewall, and then reflect off the surface, for example, at 200$400-\mathrm{km}$ radius and heights below $9 \mathrm{~km}$. There are also waves in the upper part of the domain, as in the previous figures.

At model sunset, as in Fig. 11a, $\theta^{\prime}$ in the eyewall remains positive from the prior heating. The secondary 


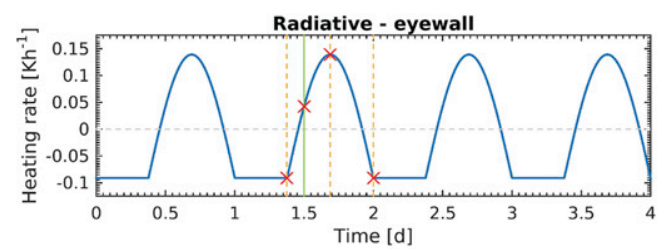

(a)

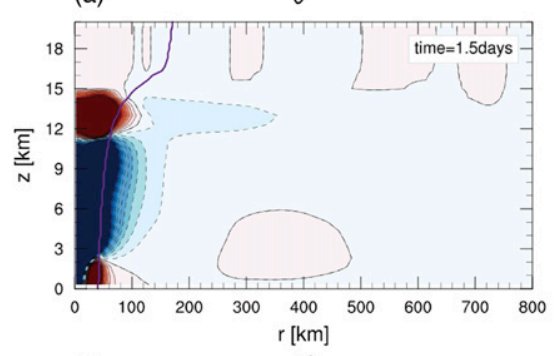

(c)

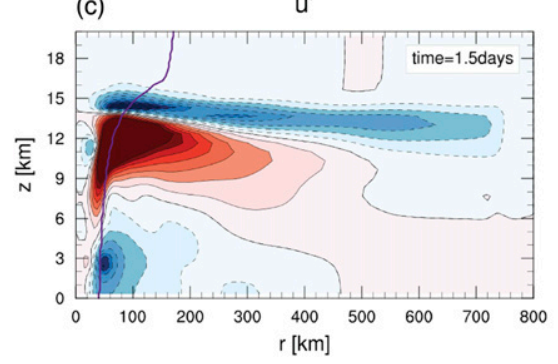

(b)
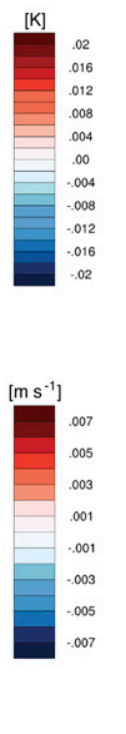

(d) w'
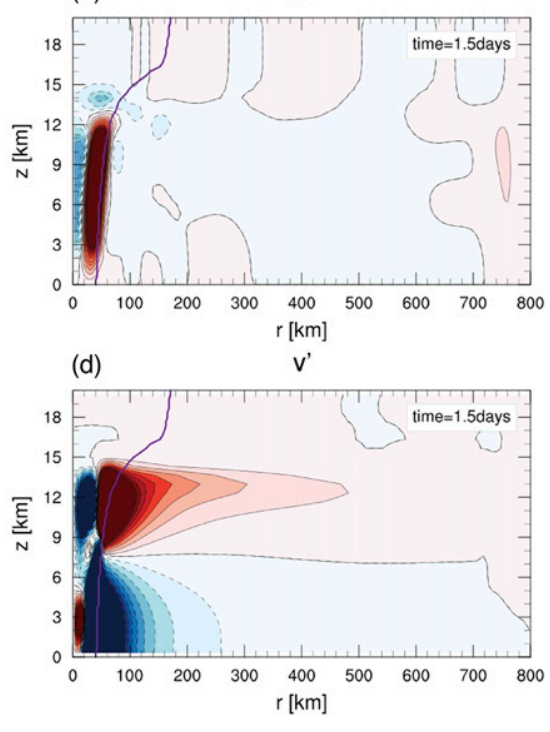
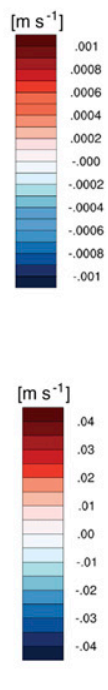

FIG. 9. As in Fig. 8, but for time $=1.500$ days, between sunrise and the heating maximum.

circulation has responded to the change to prescribed cooling, with a large counterclockwise circulation below 14-km height, as shown in Figs. 11b and 11c. There is a remnant of positive $u^{\prime}$ in at heights above $14 \mathrm{~km}$, as $\bar{v}$ is weaker here than in the lower levels, so the secondary circulation persists for longer after the change in sign of heat forcing. The $v^{\prime}$ is in the process of responding to the change in sign of heat forcing, becoming negative in the upper levels and positive in the lower levels outside the eyewall. By this time, the response is largely balanced again, since the waves shown in Fig. 9b have now radiated to the edge of the domain and been damped.

The presence of waves in the cyclone illustrates how radiative heating produces waves of higher frequency than $1 \mathrm{day}^{-1}$. For radiative heating, the temporal forcing function is not a purely sinusoidal wave, and is instead a truncated sinusoid, since it has sharp inflection points at sunrise and sunset. As such, the temporal forcing function for radiative heating projects on to higher-frequency modes, as discussed in Kossin (2002). Accordingly, higher-frequency waves are produced at sunrise and sunset. The projection on to high-frequency modes is shown in the power spectra shown in Fig. 12. The top panel shows the power spectrum for moist convective heating in the eyewall.
This has only one peak at $1 \mathrm{day}^{-1}$, since it is a purely sinusoidal wave of diurnal frequency. The bottom panel shows the power spectra for radiative heating in the eyewall and cirrus canopy. This forcing function is not sinusoidal and so projects on to many higherfrequency modes, meaning the radiative heating forcing can produce waves of higher frequency than 1 day $^{-1}$. Since this is a linear model, any waves produced in response to heating have the same frequencies as the temporal heating rate function with which they were forced. Therefore the higher-frequency components of the temporal forcing create waves that can radiate beyond the RMW.

Since the inertial stability varies as a function of $r$ and $z$, waves of different frequencies are able to radiate at different locations within the cyclone. The region in which waves can radiate varies by wave frequency is shown in Fig. 13. In Fig. 13, the radiation region occupies heights above and radii outside the line for that frequency. The radiation region grows in size with increasing frequency; thus, higher-frequency waves can radiate in a larger proportion of the cyclone than lower-frequency waves. This is consistent with the result of O'Neill et al. (2017). Low-frequency components of the power spectra, such as diurnal and semidiurnal components, are balanced in the majority of the 


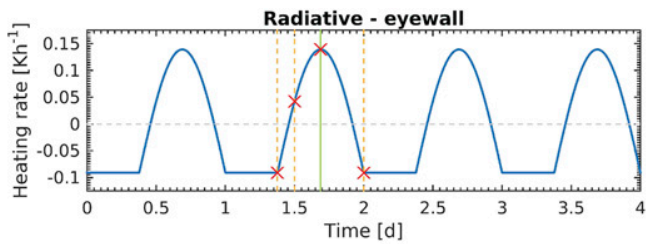

(a)

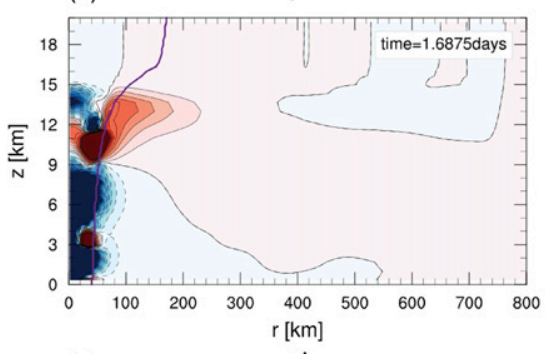

(c)

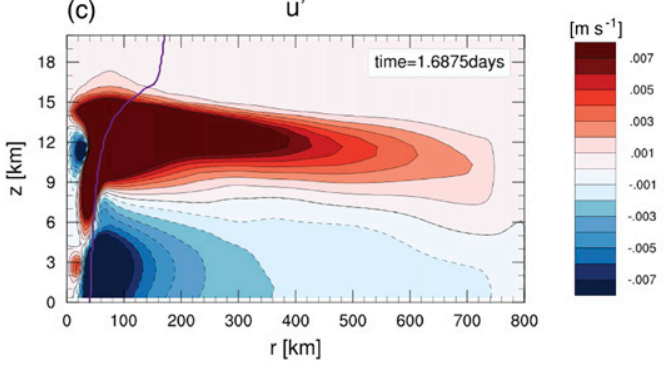

(b)

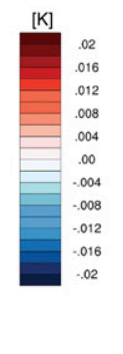

18
15
$\bar{E}_{N}$




3
0 $w^{\prime}$

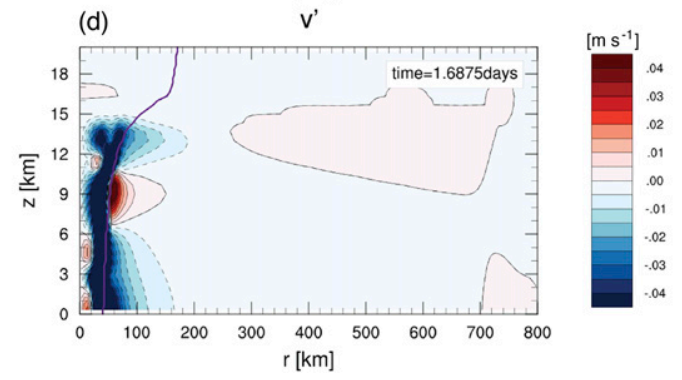

FIG. 10. As in Fig. 8, but for time $=1.6875$ days, the heating maximum.

cyclone. For example, waves of diurnal frequency at the height of the cirrus canopy $(13 \mathrm{~km})$ can only begin to radiate at radii beyond $500 \mathrm{~km}$. Therefore, as with moist convective heating in the eyewall, the response to radiative heating in the eyewall is almost entirely balanced, with some radiation of high-frequency waves. Cyclical heating and cooling in the eyewall thus produces an almost entirely balanced response that is mostly confined to inside the RMW by the high inertial stability, regardless of the shape of temporal forcing function.

\section{c. Radiative heating in the cirrus canopy}

For the simulation replicating radiative heating in the cirrus canopy, the cirrus canopy-like potential temperature tendency is forced to heat and cool according to the red line in the bottom-right panel of Fig. 4. The dynamical responses are shown in Figs. 14-17. As before, snapshots are taken at sunrise, between sunrise and maximum heating, maximum heating, and sunset.

At model sunrise, in Fig. 14a, $\theta^{\prime}$ in the cirrus canopy is negative, below which is a positive anomaly, centered at $10-\mathrm{km}$ height just outside the RMW. The cooling in the cirrus canopy induces a counterclockwise secondary circulation outside the RMW at that height, as evident in Figs. $14 \mathrm{~b}$ and $14 \mathrm{c}$. This circulation extends to the edge of the domain. There is some accumulation of wave amplitude at the edge of the domain, but no waves are reflected owing to the presence of the Gaussian damping zone. In Fig. 14b, there is downward motion in the cirrus canopy, and upward motion in the eye, outer radii at the height of the cirrus canopy, and at the edge of the domain. There is also a positive $w^{\prime}$ at heights below $9 \mathrm{~km}$, and radii beyond $360 \mathrm{~km}$, that slopes outward and downward. This is due to waves that were created at the previous sunset because of the sudden change in heating rate, the same mechanism discussed previously. These waves move radially outward from the cirrus canopy over the course of the model night. In Fig. 14c, $u^{\prime}$ converges above the cirrus canopy, with radially inward motion at heights above $12 \mathrm{~km}$ outside the RMW and outward motion just inside the RMW at that height. Below the cirrus canopy, motion is convergent, with outward motion at heights below $12 \mathrm{~km}$, and inward inside the RMW. At this time, $v^{\prime}$ is strongly negative below the cirrus canopy, and positive in the upper eye and upper-cirrus canopy, as shown in Fig. 14d.

This spatial pattern of $\theta^{\prime}$ and $v^{\prime}$ persists for a few hours after model sunrise, as shown in Fig. 15, between sunrise and peak heating. The $\theta^{\prime}$ and $v^{\prime}$ fields have not yet responded to the change from prescribed cooling to 

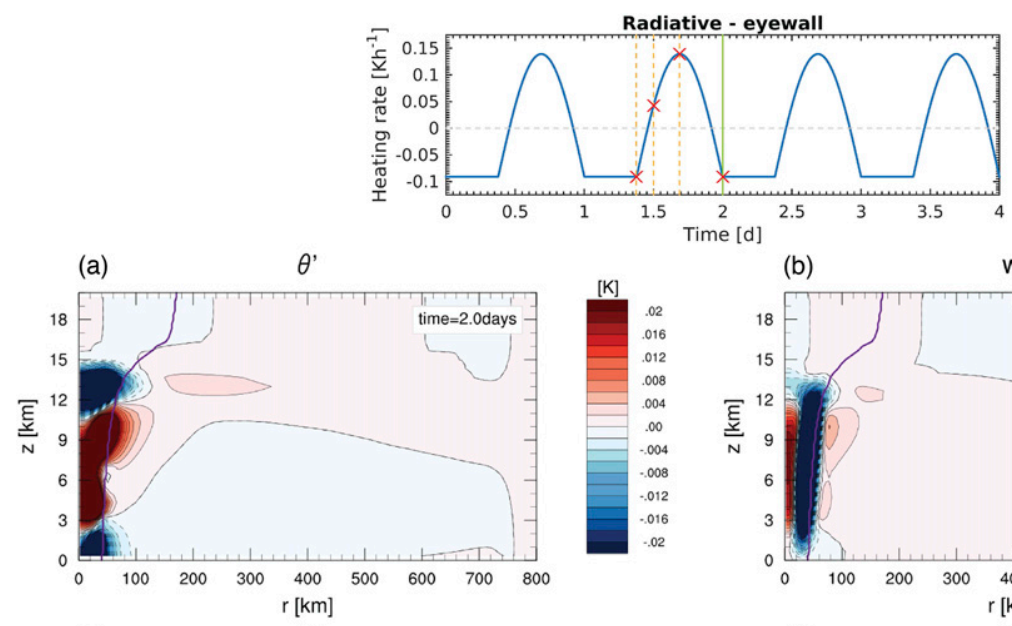

(b)
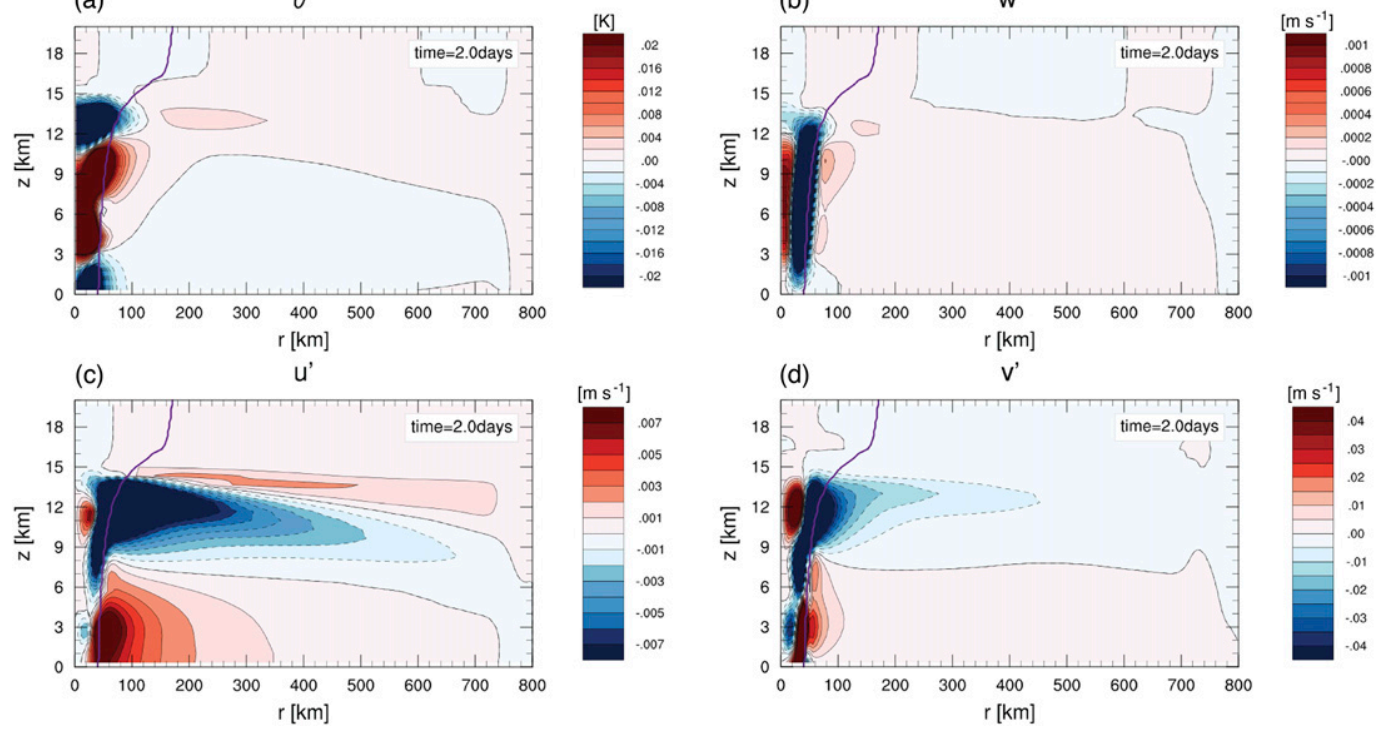

(d)

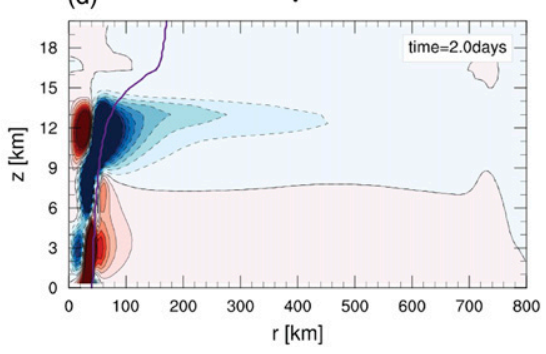

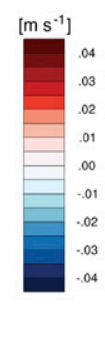

FIG. 11. As in Fig. 8, but for time $=2.000$ days, the model sunset.

heating. The secondary circulation is responding to the change to positive heating rate. While the spatial pattern of $u^{\prime}$ has largely persisted outside the RMW, inside the RMW the circulation has become counterclockwise. There is strong negative $w^{\prime}$ in the upper eye, and weak upward motion at radii beyond $350 \mathrm{~km}$ at the height of the cirrus canopy. Furthermore, there is a positive $w^{\prime}$ feature extending from the cirrus canopy downward, at radii beyond the RMW. These are the high-frequency waves that were created at sunrise by the sudden change in heating rate. Waves of higher frequency radiate at a steeper angle from the horizontal than those of lower frequency.

By the peak heating, as in Fig. 16, $\theta^{\prime}$ in the cirrus canopy has begun to respond to the prescribed heating, more so than in the eyewall cases. This is because the cirrus canopy inertial stability is lower, since it is located at a greater radius and lower $\bar{v}$ than the eyewall, permitting a quicker response of $\theta^{\prime}$ to the change in prescribed heating. The inertial stability dictates the magnitude of $u^{\prime}$, and therefore radial advection of $\bar{\theta}$. As shown in Eq. (1e), the tendency of $\theta^{\prime}$ is dependent on this radial advection. As such, where inertial stability is low, $u^{\prime}$ can be large, causing $\theta^{\prime}$ to respond more quickly. The maximum $\theta^{\prime}$ is at $12-15-\mathrm{km}$ height, extending to a radius of around $420 \mathrm{~km}$.
Underlying this positive $\theta^{\prime}$ is a negative anomaly at 9$12-\mathrm{km}$ height. By this time, the secondary circulation has reversed, becoming clockwise outside the RMW. In the cirrus canopy, $w^{\prime}$ is positive, and is negative in the upper eye and outer radii at the height of the cirrus canopy. As before, there is a band of positive $w^{\prime}$ radiating outward and downward from the cirrus canopy, and there are waves at the surface. Above the cirrus canopy, $u^{\prime}$ is divergent, with outward $u^{\prime}$ beyond the RMW and inward $u^{\prime}$ inside the RMW. Below the cirrus canopy, $u^{\prime}$ is convergent. There is a remnant of negative $u^{\prime}$ from radii of $550-740 \mathrm{~km}$, and heights of $12-15 \mathrm{~km}$. This is because this area is displaced far from the forcing, and thus takes longer to respond than the inner radii. In response to the daytime heating, the negative $v^{\prime}$ has moved upward and the positive $v^{\prime}$ in the cirrus canopy has dissipated. As with $u^{\prime}$, there is a remnant positive $v^{\prime}$ at radii beyond $360 \mathrm{~km}$ at the level of the cirrus canopy. As shown in Eq. (1b), the tendency in $v^{\prime}$ depends on the radial and vertical gradients of $\bar{v}$. The relative vorticity $[(1 / r)(\partial r \bar{v} / \partial r)]$ decreases rapidly to a very small value outside the RMW, and is not strongly dependent on $r$ outside the RMW. At the height of the cirrus canopy, $\partial \bar{v} / \partial z$ is strongly dependent on $r$, with a magnitude at the RMW 2-4 times that at the edge of 


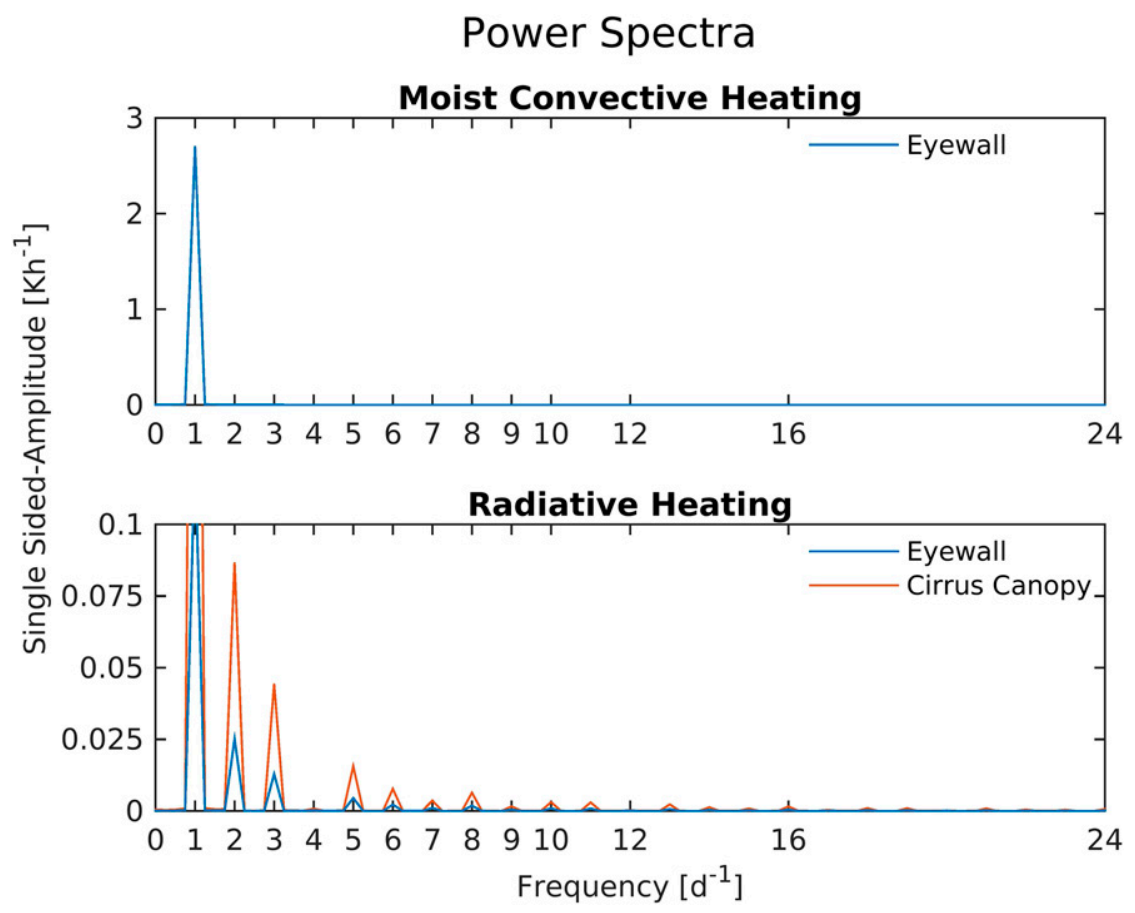

FIG. 12. Power spectra of the temporal forcing functions in the right panels of Fig. 4. The frequencies are shown here as the number of waves per day, where $1 \mathrm{day}^{-1}$ is the diurnal frequency $\left(7.29 \times 10^{-5} \mathrm{~s}^{-1}\right), 2 \mathrm{day}^{-1}$ is the semidiurnal frequency $\left(14.58 \times 10^{-5} \mathrm{~s}^{-1}\right)$, and so on. (top) Power spectrum for moist convective heating in the eyewall. (bottom) Power spectra for radiative heating in the eyewall (blue) and cirrus canopy (red). The $y$ axis is limited at $0.1 \mathrm{~K} \mathrm{~h}^{-1}$ to show the very small amplitude of the high-frequency components.

the domain. Therefore, the response of $v^{\prime}$ is significantly slower in the outer cyclone because it is farther from the forcing, and because the magnitude of $\partial \bar{v} / \partial z$ is lower.

At model sunset, as in Fig. 17, the cirrus canopy has begun to respond to the prescribed cooling, with the positive $\theta^{\prime}$ weakening in the location of the forcing. The $w^{\prime}$ component of the secondary circulation has essentially returned to that of sunrise, but $u^{\prime}$ has not fully returned to the spatial pattern at sunrise, as there is a remnant of positive $u^{\prime}$ at radii beyond $200 \mathrm{~km}$, and height of $12-15 \mathrm{~km}$. This again is due to the long lag in response at this distance from the forcing. By this time, many of the high-frequency waves that were created at sunrise have radiated almost to the edge of the domain. At the same time, $v^{\prime}$ is still responding to the change to cooling, with a weak negative anomaly persisting in the cirrus canopy from the earlier heating. The negative anomaly below the cirrus canopy is much lower in amplitude by this time, having not fully dissipated during the daytime heating. Thus throughout the day, there is a very weak and consistent negative $v^{\prime}$ at the surface. It should be noted that this negative $v^{\prime}$ does not weaken the basic-state vortex, since in a linear model anomalies are not permitted to change the basic state.

In Figs. 14-17, below 6-km height $\theta^{\prime}$ remains positive, and below $8 \mathrm{~km} v^{\prime}$ remains negative, throughout the entire $24 \mathrm{~h}$ of the model day. The consistent presence of the $\theta$ anomaly could potentially be because it is displaced a considerable vertical distance from the cirrus canopy heat forcing, and therefore the oscillation of the prescribed heating in the cirrus canopy would have to be slower than one day for the anomalous cirrus canopy heating to affect the lower cyclone. It is suggested here that the sign of the anomaly is positive because the temporal forcing function is negative for slightly more of the day than it is positive, meaning the cirrus canopy is cooled for a greater proportion of any given day than it is heated. Therefore, the lower-level positive anomaly, created during the cirrus canopy cooling, can persist throughout the day as the daytime upper-level heating does not last proportionally long enough for the lower-level anomaly to dissipate, even in the presence of time-mean zero forcing. This does not appear to be a result of the initial condition, since this also occurs when the simulation begins at different times of 


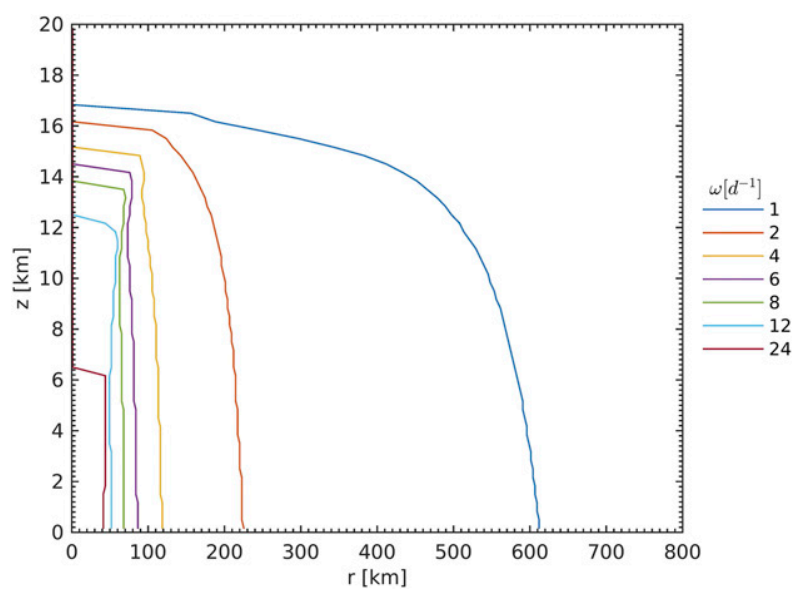

FIG. 13. Regions in which gravity waves can radiate by frequency. The frequencies are shown here as the number of waves per day, where 1 day $^{-1}$ is the diurnal frequency $\left(7.29 \times 10^{-5} \mathrm{~s}^{-1}\right)$, 2 day $^{-1}$ is the semidiurnal frequency $\left(14.58 \times 10^{-5} \mathrm{~s}^{-1}\right)$, and so on. Waves of any given frequency are stable in the region outside the line for that frequency (at heights above and radii beyond the line).

day. This positive anomaly usually forms after the simulation has completed one full model night (not shown). The nonzero mean $\theta^{\prime}$ and $v^{\prime}$ is likely due to diffusion, but could alternately be an example of diurnal rectification as outlined in Cronin et al. (2015), where time-mean zero oscillatory forcing can produce a response with nonzero time mean.

It is clear from $w^{\prime}$ that radiative heating of the cirrus canopy produces a response very different in character to radiative heating of the eyewall, in that radiation of waves is much more prevalent. For the eyewall, the vast majority of the response was balanced and confined to the region inside the RMW, whereas for the cirrus canopy, the part of the heat forcing that excites high frequencies produces a radiating response outside $100-\mathrm{km}$ radius. This is likely since much of the cirrus canopy is located outside the RMW, and therefore much of the balanced and radiating responses are not confined to inside the RMW. Therefore, twice per day, at the inflection points in the forcing corresponding to sunrise and sunset, a group of high-frequency waves is created that can radiate throughout the cyclone. In a more realistic tropical cyclone, this would indicate that high-frequency waves created at sunrise and sunset are more likely to originate in the cirrus canopy, as opposed to the eyewall, since waves created in the eyewall are less prone to escape the RMW. The diurnal oscillation in the cirrus canopy is balanced to large radii at the height of the cirrus canopy. The alternating positive and negative $w^{\prime}$ at this height is caused by alternating heating and cooling, rather than true radiation of a wave.
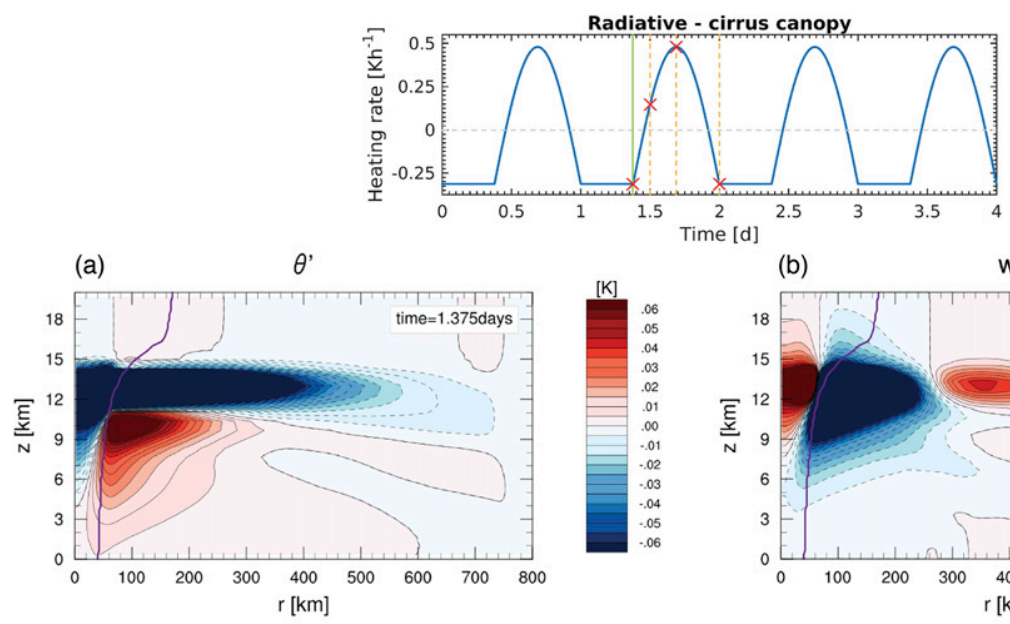

(b) $\quad$ w
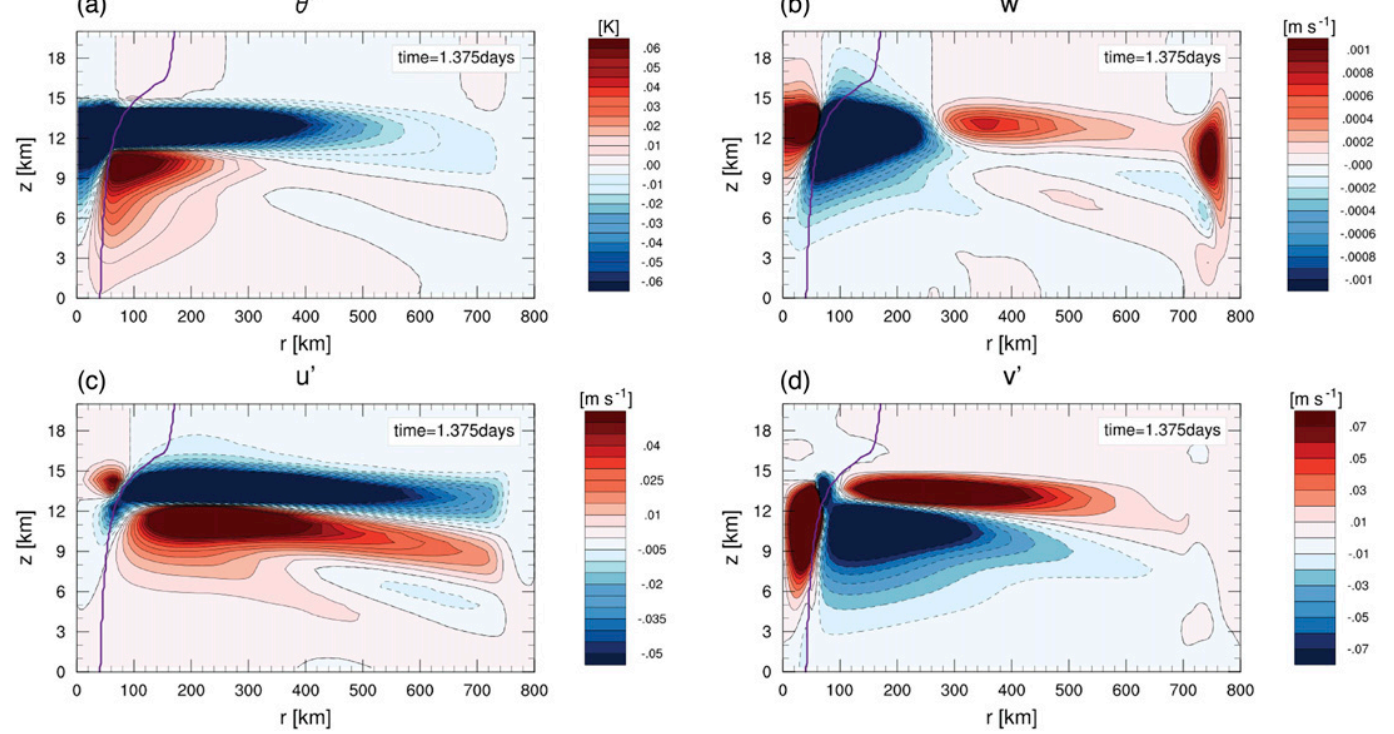

(d)

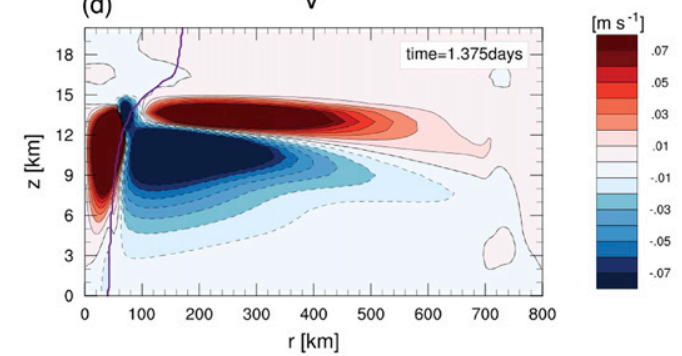

FIG. 14. Radiative heating in the cirrus canopy at 1.375 days, the model sunrise. $r-z$ snapshots of (a) $\theta^{\prime}$ (K), (b) $w^{\prime}$ $\left(\mathrm{m} \mathrm{s}^{-1}\right),(\mathrm{c}) u^{\prime}\left(\mathrm{m} \mathrm{s}^{-1}\right)$, and $(\mathrm{d}) v^{\prime}\left(\mathrm{m} \mathrm{s}^{-1}\right)$. The saturation of the color scale, and the line and marker colors are as in Fig. 5. 


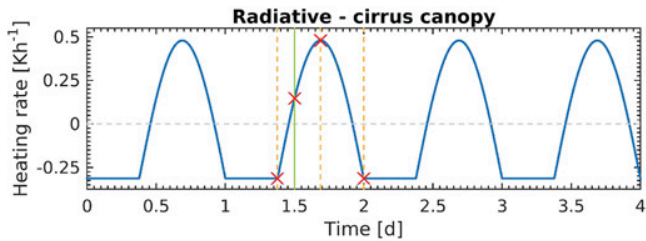

(a)

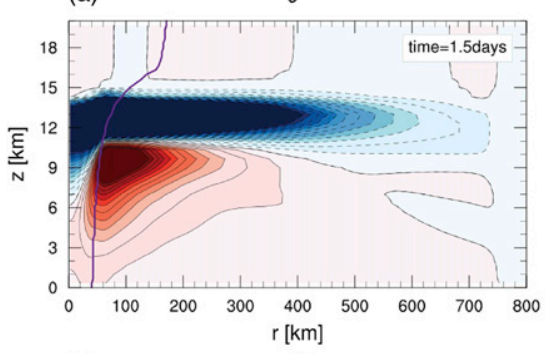

(c)

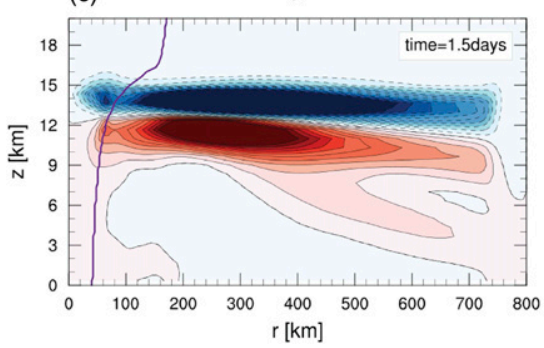

(b)
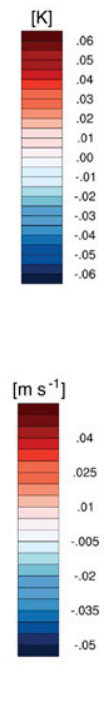

$w^{\prime}$

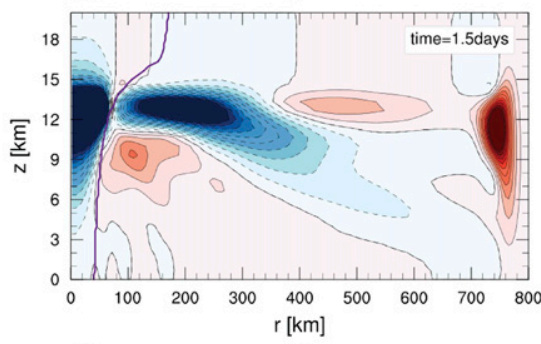

v'

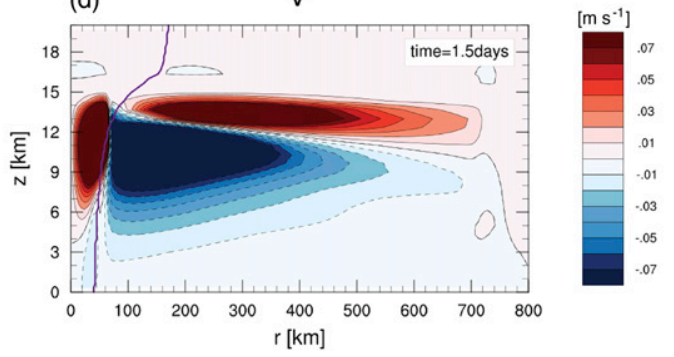

FIG. 15. As in Fig. 14, but for time $=1.500$ days, between sunrise and the heating maximum.

This is illustrated in Fig. 18, which shows a Hovmöller diagram of $w^{\prime}$ at a height of $13.33 \mathrm{~km}$, approximately the height of the vertical center of the model cirrus canopy. In Fig. 18a, during the model nighttime, downward motion is produced in the vicinity of the forcing, between $60-$ and $280-\mathrm{km}$ radius. There is compensating upward motion both inside $60 \mathrm{~km}$ and outside $280 \mathrm{~km}$. The radius at which $w^{\prime}$ changes sign will be set by the size of the forcing and the Rossby radius of deformation. During the model daytime, the radial pattern of $w^{\prime}$ reverses. Since the temporal forcing is negative for a greater proportion of the day than it is positive, the upward motion between 60 and $280 \mathrm{~km}$ occurs for a shorter time than the nighttime downward motion. The $w^{\prime}$ induced between 60 and $200 \mathrm{~km}$ in the response to heat forcing does not appear to radiate outward, and instead is a stationary balanced response. The change in sign of $w^{\prime}$ between 250 and $290 \mathrm{~km}$ lags behind the change in sign of the heat forcing, so that when heat forcing is zero there is still remnant $w^{\prime}$. Between $280-$ and $400-\mathrm{km}$ radius, there is a weak alternating positive and negative $w^{\prime}$ that does not appear to radiate outward, and is instead also a stationary balanced response. However, there is evidence of radiating waves outside $280-\mathrm{km}$ radius. In Fig. 18b, the Hovmöller is restricted to radii between 250 and $600 \mathrm{~km}$, and is saturated at $20 \%$ of maximum $w^{\prime}$. From the initiation of the model until 0.5 days in to the simulation, there is evidence of rapid adjustment to onset of the forcing, with radiating waves giving the contours a serrated appearance. Radiation of high-frequency waves at these radii is also evident later in the simulation when $w^{\prime}$ is positive, during the model night, as the high-frequency waves that were created at sunset radiate outward. The outward radiating feature at radii beyond $270 \mathrm{~km}$ is likely not a wave of diurnal frequency, but is instead the balanced diurnal response and wave packets of higher-frequency waves created at sunrise and sunset.

\section{d. Sensitivity simulations}

To determine the impact of stratification on the results shown above, simulations not shown here were also performed with $N^{2}$ set to a constant value of $0.5 \times$ $10^{-4}, 1 \times 10^{-4}$, or $2 \times 10^{-4} \mathrm{~s}^{-2}$ throughout the domain. Additional simulations were performed with constant $N^{2}$ of $1 \times 10^{-4} \mathrm{~s}^{-2}$ to a height of $12 \mathrm{~km}$, above which $N^{2}$ linearly increases to a maximum of $4 \times 10^{-4} \mathrm{~s}^{-2}$ at the top of the domain. For the cases with constant $N^{2}$, the dynamical responses were almost exactly the same as those with realistic stratification, with the exception of 


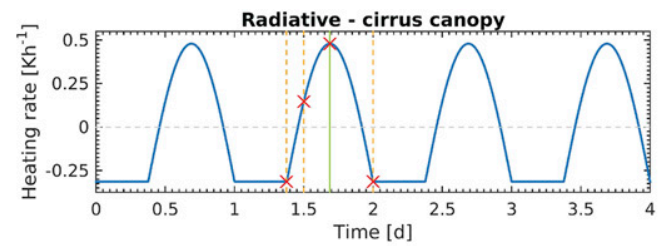

(a)

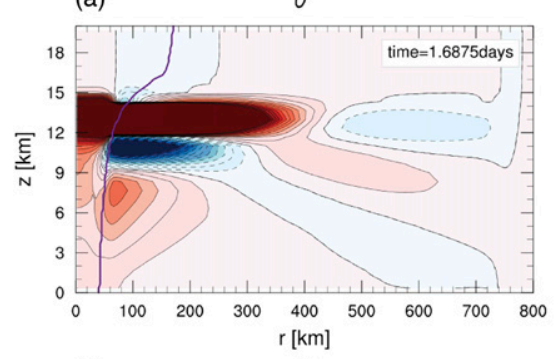

(c)

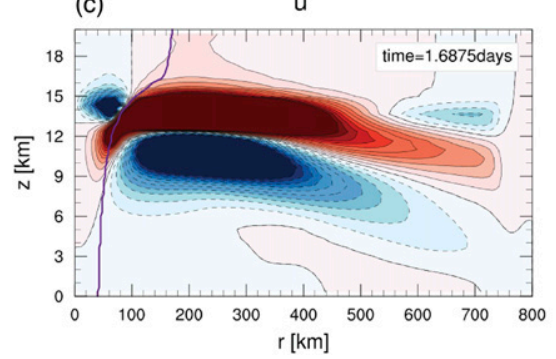

(b)
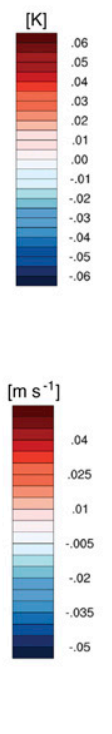

(d) w'
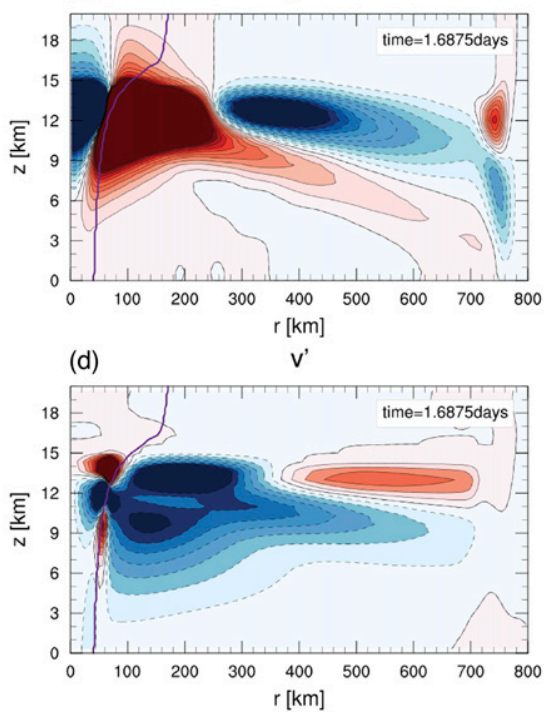
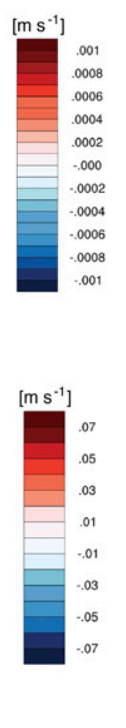

FIG. 16. As in Fig. 14, but for time $=1.6875$ days, the heating maximum.

very small differences in the uppermost parts of the domain. For example, the negative anomaly at $600-\mathrm{km}$ radius and 13-km height in the $w^{\prime}$ panel of Fig. 17 extends to the top of the domain when stratification is constant. This is likely because the waves can radiate as easily upward as they can downward when stratification is constant. Other than some extremely small differences in the uppermost part of the domain, the dynamical response was exactly the same as for the realistic stratification simulations. For the case with constant $N^{2}$ in the lower $12 \mathrm{~km}$, and increasing $N^{2}$ above $12 \mathrm{~km}$, the dynamical response is again extremely similar to that of the simulations with realistic stratification. In this case, high wave amplitude that accumulates at the edge of the domain was found to be slightly closer to the edge of the domain, but the waves still did not reflect. The effect of changes in stratification on the dynamical response of a TC to diurnal heating in 3DVPAS are therefore considered negligible.

The dynamic viscosity used in the simulations shown here is $40 \mathrm{~m}^{2} \mathrm{~s}^{-1}$. However, other simulations not shown here used values of 20 and $10 \mathrm{~m}^{2} \mathrm{~s}^{-1}$; the value chosen had a negligible effect on the result. For the simulation with a dynamic viscosity of $10 \mathrm{~m}^{2} \mathrm{~s}^{-1}$, the results were very similar to those with $40 \mathrm{~m}^{2} \mathrm{~s}^{-1}$, with the exception of the appearance of noise. Although also not shown, the impact of the Coriolis parameter $f$ on the results above was also found to be negligible. The simulations in section 3 were also performed with an $f=0 \mathrm{~s}^{-1}$, which yielded a very similar dynamical response as those with an $f$ corresponding to $20^{\circ} \mathrm{N}$.

The regions in which gravity waves can radiate are dictated by inertial stability, and thus are highly sensitive to the setup of the vortex. The radiation regions in Fig. 13 were also calculated with varying vortex properties, such as the strength of the wind maximum, and the location of the radius of maximum wind. To test the effect of these on the radiation regions, we used the following vortices: wind maximum of $45 \mathrm{~m} \mathrm{~s}^{-1}$ with the RMW at $40 \mathrm{~km}$ (the vortex in the simulations shown here), $45 \mathrm{~m} \mathrm{~s}^{-1}$ at $80 \mathrm{~km}, 22.5 \mathrm{~m} \mathrm{~s}^{-1}$ at $40 \mathrm{~km}$, and $22.5 \mathrm{~m} \mathrm{~s}^{-1}$ at $80 \mathrm{~km}$. Increasing the RMW increases the radius at which waves of a given frequency can begin to radiate and decreases the height (i.e., the lines move out and down). For example, at $45 \mathrm{~m} \mathrm{~s}^{-1}$, a wave of frequency 2 day $^{-1}$ at the surface begins to radiate $60 \mathrm{~km}$ farther out when the RMW is doubled. Furthermore, waves of frequency 24 day $^{-1}$ can radiate in the entire cyclone, even in the lower eye and eyewall, when the RMW is doubled. Weakening the 


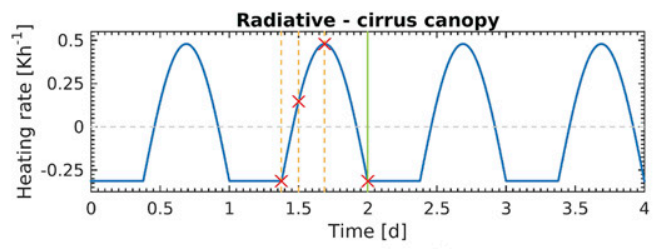

(a)

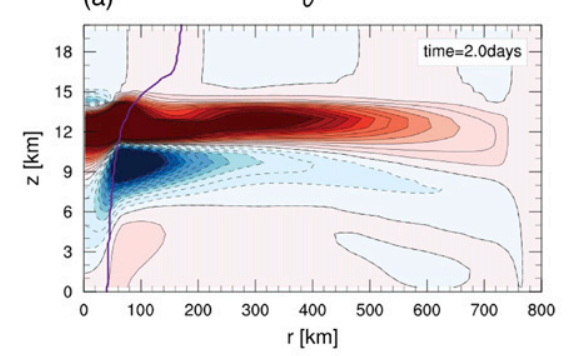

(c)

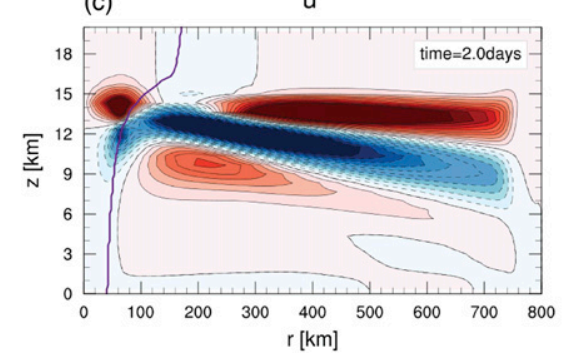

(b)
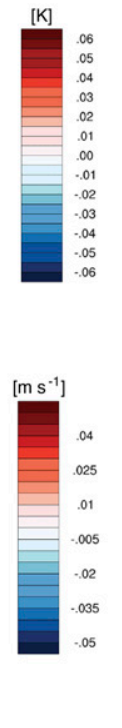

w'
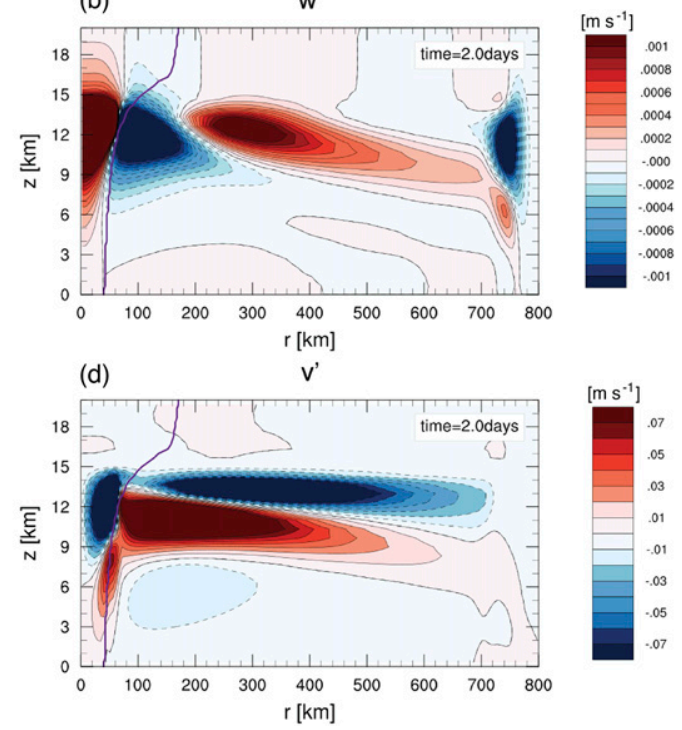

FIG. 17. As in Fig. 14, but for time $=2.000$ days, the model sunset.

wind maximum also strongly decreases the radius at which waves can begin to radiate. For example, for a wind maximum of $22.5 \mathrm{~m} \mathrm{~s}^{-1}$ at an RMW of $40 \mathrm{~km}$, waves of frequency 2 day $^{-1}$ begin to radiate $80 \mathrm{~km}$ farther inward than for a wind maximum of $45 \mathrm{~m} \mathrm{~s}^{-1}$ at the same RMW. As before, at this wind speed, doubling the RMW increases the radius at which a wave of any given frequency can begin to radiate, and decreases the height at which radiation becomes possible. The radiation regions begin at greater radii in this study than in O'Neill et al. (2017) since our vortex is much broader.

\section{Discussion and conclusions}

Motivated by the findings of Dunion et al. (2014), this study used idealized simulations to determine how the diurnal cycle manifests itself in balanced and radiating responses in tropical cyclones, and to study the extent to which linear dynamics can capture the DC in
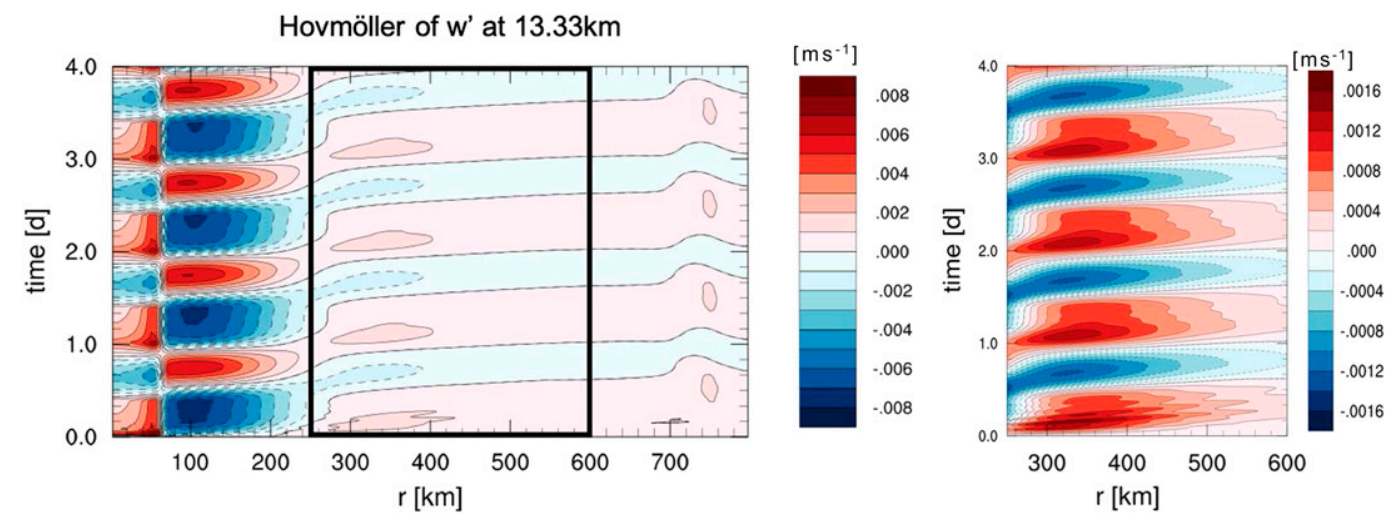

FIG. 18. Hovmöller of $w^{\prime}\left(\mathrm{m} \mathrm{s}^{-1}\right)$ at a height of $13.33 \mathrm{~km}$ from radii (a) between 0 and $800 \mathrm{~km}$ and (b) between 250 and $600 \mathrm{~km}$. The black box in (a) denotes the region plotted in (b). The color scale in (b) is saturated at $20 \%$ of the maximum $w^{\prime}$ to show finer detail of the motion. 
TCs. A linear dynamical model, 3DVPAS, was forced with potential temperature tendencies aiming to replicate the diurnal cycles of heating in the cirrus canopy and eyewall of TCs that we see in a more realistic model. The main sources of diurnal heating in a $\mathrm{TC}$ are moist convective heating of the eyewall, and radiative heating of the cirrus canopy and eyewall. We used the shortwave, longwave, and moist convective heating tendencies from the Nolan et al. (2013) hurricane nature run as physical motivation for the forcing in 3DVPAS.

The results showed that while some waves that originated in the eyewall were able to radiate beyond the RMW, it was much easier for responses cause by heating in the cirrus canopy to radiate throughout the cyclone. This illustrated that the location of the application of diurnal heating has a strong impact on the dynamical response, and that the nonsinusoidal nature of the DC of radiative heating results in the production of high-frequency gravity waves. The low-frequency components of the DC therefore project on to a balanced response throughout the majority of the cyclone, whereas the high-frequency components project on to gravity waves that radiate outward and downward from the location of the forcing. Moist convective and radiative diurnal heating in the eyewall produce a predominantly balanced response in 3DVPAS, whereby the $w^{\prime}$ response is confined to the region near the source by the high inertial stability. When diurnal heating is applied in the eyewall, a secondary circulation is induced throughout the entire depth of the cyclone. Radiative diurnal heating in the cirrus canopy produces both balanced and radiating responses throughout the depth of the cyclone, since the source of the heating is outside the RMW. The secondary circulation that is produced in response to cirrus canopy heating is largely confined to the upper levels, in the vicinity of the forcing.

The responses to heating have the same frequencies as the forcing. Higher-frequency waves can begin to radiate at a smaller radius than those of lower frequency. Diurnal-frequency waves can therefore only begin to radiate at large radii. This is strongly dependent on the basic-state vortex, as in a narrower vortex, radiating waves of diurnal frequency could exist at smaller radii. Packets of higher-frequency waves are produced twice per day, at model sunrise and sunset, because of the relatively sudden change in heating rate. The results presented here suggest that diurnal signals beyond the inner core of a TC originate as a result of diurnal forcing of the cirrus canopy as opposed to the eyewall.

The results presented in this study compare well with previous studies of the DC in the cirrus canopy using full-physics simulations in axisymmetric and threedimensional frameworks (Navarro and Hakim 2016; Navarro et al. 2017; O'Neill et al. 2017). O'Neill et al. (2017) used the Khairoutdinov and Randall (2003) system for atmospheric modeling (SAM), which incorporates full physics, to study the transition between balanced and radiating responses to diurnal heating in the cirrus canopy. They found that waves can only begin to propagate well beyond the storm core, and that higher-frequency waves can begin to propagate closer to the storm core. Navarro and Hakim (2016) and Navarro et al. (2017) performed a simulation of the DC in the cirrus canopy in an idealized full-physics model, treating diurnal forcing as a sine wave. The idealized model is based on a version of the SawyerEliassen equation modified to allow for time-varying forcing. The secondary circulation response in our 3DVPAS simulations compares well with their result, with the exception that our forcing induces waves of higher frequency than $1 \mathrm{day}^{-1}$, since our forcing is not purely sinusoidal. Navarro et al. (2017) validated their results against the Bryan and Fritsch (2002) Cloud Model 1 (CM1), finding strong qualitative similarities. The vertical motion response to heating in their study is an order of magnitude larger than that shown here. For nonrotating and TC environments, respectively, Ruppert and Hohenegger (2018) and Ruppert and O'Neill (2019) illustrated that the DC of solar radiation causes a two-layered response: a nighttime enhancement of eyewall convection in the low to midlevels, and a daytime lifting of the cloud anvil. The results of the linear dynamics presented here support their suggestion that daytime radiative heating directly causes upward motion, thereby lifting the cloud anvil.

Based on how well our results compare with these studies, the lack of nonlinearity in 3DVPAS does not appear to be problematic in capturing the essential behavior of the DC in TCs. The 3DVPAS can therefore yield a useful result in diagnosing balanced versus radiating responses to diurnal heating in axisymmetric TCs. Furthermore, these results illustrate that while most observations of the DC in TCs are of upper-level clouds, responses to diurnal heating in TCs are present throughout the entire cyclone. However, since these results are very idealized, the extent to which they apply to real TCs and asymmetric storms is unclear.

This work raises interesting questions about the impact of waves produced by diurnal heating, in particular those radiating from the cirrus canopy. Based on the results presented here, diurnal heating in the eyewall, whether due to moist convection or radiation, induces diurnal variability in the tangential wind maximum, and weak variability in upper-level 
outflow. This diurnal variability in lower to midlevel $v^{\prime}$ in the inner core could be of particular interest since a recent study by Tang et al. (2019) illustrated that vertical advection of tangential wind tendency is enhanced overnight. Diurnal heating in the cirrus canopy has little effect on surface tangential wind, but induces waves that can radiate easily throughout the cyclone. It is possible, in a nonlinear framework that permits convection, that these waves could be of high enough amplitude to induce anomalous lifting and precipitation on diurnal time scales. This feature has been noted in Dunion et al. (2019), who describe a diurnal squall line at outer radii in the HNR. This could potentially be caused by waves radiating from the cirrus canopy, or balanced responses on lowfrequency time scales. The impact of the dynamical responses to diurnal heating in TCs, and the extent to which nonlinearity is important should be addressed in future work.

Acknowledgments. The authors extend thanks to the extremely helpful reviews by the three reviewers for this article. This research was supported by the National Science Foundation under Award AGS-1654831.

\section{APPENDIX}

\section{A Mathematical Description of the Heat Forcing in 3DVPAS}

Equation (A1) describes the cirrus canopy-like heat forcing, which produces the spatial heating rate shown in Fig. 3a:

$$
\begin{aligned}
& \dot{Q}_{\theta}\left(r<r_{\text {center }}, z, t_{0}\right) \\
& \quad=\dot{\theta}_{0} \exp \left[-\left(\frac{r-r_{\text {center }}}{\sigma_{r}}\right)^{2}-\left(\frac{z-z_{\text {center }}}{\sigma_{z}}\right)^{2}\right], \\
& \dot{Q}_{\theta}\left(r \geq r_{\text {center }}, z, t_{0}\right) \\
& \quad=\dot{\theta}_{0} \exp \left[-\left(\frac{r-r_{\text {center }}}{\sigma_{r}}\right)^{2}-\left(\frac{z-z_{\text {center }}}{\sigma_{z} \Xi}\right)^{2}\right],
\end{aligned}
$$

where $\dot{Q}_{\theta}$ is the potential temperature forcing, and $\dot{\theta}_{0}$ is the maximum heating rate at the initial time $t_{0}$. At time $t_{0}, \dot{\theta_{0}}$ is equal to the heating rate at 00:00 for the respective simulation in the right panels of Fig. 4. The $\dot{\theta}$ then evolves with time according to the temporal forcing equations outlined below. The $r$ and $z$ are the radial and vertical coordinates, respectively. The $r_{\text {center }}$ and $z_{\text {center }}$ are the radial and vertical coordinates of the maximum heating rate in the cirrus canopy (the center of the canopy). The $z_{\text {center }}$ is $13 \mathrm{~km}$, and $r_{\text {center }}$ is $72 \mathrm{~km}$, which is equal to the radius of maximum wind at $z_{\text {center }}$. The $\sigma_{r}$ and $\sigma_{z}$ are the radial and vertical half widths of the Gaussian region; $\sigma_{z}$ is equal to $0.8 \mathrm{~km}$. The $\sigma_{r}$ is equal to $11 \mathrm{~km}$ for the region radially inward of $r_{\text {center }}$ [Eq. (A1a)], and is equal to $150 \mathrm{~km}$ for the region radially beyond $r_{\text {center }}$ [Eq. (A1b)]. To imitate the thinning of the cirrus canopy with increasing radius, the potential temperature anomaly is made to contract in vertical extent with increasing radius from $r_{\text {center. }}$. This is achieved through the "thickening factor," denoted by $\Xi$. The value of $\Xi$ decreases linearly with radius, from a value of 1 at $r_{\text {center }}$ to a value of 0.6 at the edge of the domain, such that the cirrus canopy thins outward of the RMW.

Equation (A2) describes the eyewall-like heat forcing, which produces the spatial heating rate shown in Fig. 3b:

$$
\begin{aligned}
& \dot{Q}_{\theta}\left(r, z<2500 \mathrm{~m}, t_{0}\right) \\
& \quad=\dot{\theta}_{0} \exp \left[-\left(\frac{r-r_{\text {center }}-r_{\text {shift }}}{\sigma_{r}}\right)^{2}-\left(\frac{z-z_{\text {center }}}{\sigma_{z}}\right)^{2}\right],
\end{aligned}
$$

$$
\begin{aligned}
& \dot{Q}_{\theta}\left(r, 2500 \leq z \leq 11000 \mathrm{~m}, t_{0}\right) \\
& \quad=\dot{\theta}_{0} \exp \left[-\left(\frac{r-r_{\text {center }}-r_{\text {shift }}}{\sigma_{r}}\right)^{2}\right], \\
& \dot{Q}_{\theta}\left(r, z>11000 \mathrm{~m}, t_{0}\right) \\
& \quad=\dot{\theta}_{0} \exp \left[-\left(\frac{r-r_{\text {center }}-r_{\text {shift }}}{\sigma_{r}}\right)^{2}-\left(\frac{z-z_{\text {center }}}{\sigma_{z}}\right)^{2}\right] .
\end{aligned}
$$

The terms in Eq. (A2) are the same as those in Eq. (A1), with the addition of a new term $r_{\text {shift }}$, which describes the shift of the heating maximum to be $10 \mathrm{~km}$ inward of the RMW. In Eq. (A2a), $r_{\text {center }}$ is defined as the RMW at height $z_{\text {center }}$ of $2.5 \mathrm{~km}$. In Eq. (A2b), $r_{\text {center }}$ is defined as the RMW corresponding to height, $z$. In Eq. (A2c), $r_{\text {center }}$ is defined as the RMW at height $z_{\text {center }}$ of $11 \mathrm{~km}$. The $\sigma_{r}$ and $\sigma_{z}$ are both equal to $15 \mathrm{~km}$ in Eq. (A2).

The potential temperature perturbations are forced to evolve with time according to Eqs. (A3) and (A4) for moist convective heating and radiative heating, respectively.

Equation (A3) is sinusoidal to replicate the DC of moist convective heating in the eyewall, and produces the forcing function in the top-right panel of Fig. 4: 


$$
\dot{Q}_{\theta}(t)=\theta_{\text {amp }} \sin \left(2 \pi \frac{t+t_{\text {peak }}+12 \mathrm{~h}}{T}\right) .
$$

The $\dot{Q}_{\theta}$ is defined as before, and $\theta_{\mathrm{amp}}$ is the amplitude of the sine wave, which is here chosen to be $2.1 \mathrm{~K} \mathrm{~h}^{-1}$. This value was chosen as it is the amplitude of the DC, found by computing the power spectrum of the low-pass-filtered time series in the top-left panel of Fig. $4 ; t_{\text {peak }}$ is the time at which peak heating occurs, which is around 2100 UTC for moist convective heating in the eyewall. The $T$ is the duration of 1 day.

Equation (A4) imitates the DC of radiative heating in the cirrus canopy and eyewall, and produces the temporal forcing functions as in the bottom-right panel of Fig. 4:

$$
\dot{Q}_{\theta}(t)=\left\{\begin{array}{cc}
2 \theta_{\mathrm{SW}} \sin \left(2 \pi \frac{t-t_{\text {sunrise }}}{T_{\text {daylight }}}\right)-\theta_{\mathrm{LW}} & \text { if } \quad 0900 \leq t \leq 2330 \mathrm{UTC} \\
-\theta_{\mathrm{LW}} & \text { if } \quad t<0900 \mathrm{UTC} \text { or } t>2330 \mathrm{UTC}
\end{array} .\right.
$$

Between sunrise $t_{\text {sunrise }}$ at $0900 \mathrm{UTC}$, and sunset, at 2330 UTC, the forcing function is the sum of solar shortwave heating plus longwave cooling. The solar heating follows a sinusoidal shape throughout the day, with a maximum amplitude $\theta_{\text {Sw }}$ of $0.4 \mathrm{~K} \mathrm{~h}^{-1}$ for radiative heating of the cirrus canopy and $0.12 \mathrm{~K} \mathrm{~h}^{-1}$ for radiative heating of the eyewall. The $T_{\text {daylight }}$ denotes the length of time between sunrise and sunset, equal to $14.5 \mathrm{~h}$. The $\theta_{\mathrm{LW}}$ is the magnitude of the longwave cooling and remains constant throughout the day. From the HNR, $\theta_{\mathrm{LW}}$ is around $0.4 \mathrm{~K} \mathrm{~h}^{-1}$ for the cirrus canopy and $0.2 \mathrm{~K} \mathrm{~h}^{-1}$ for the eyewall. The temporal forcing functions in 3DVPAS were adjusted to have a mean value of zero by setting $\theta_{\mathrm{LW}}$ to the mean value of $\theta_{\mathrm{SW}}$. Since there is no solar shortwave heating overnight, the forcing function from sunset to sunrise is simply the cooling due to $\theta_{\mathrm{LW}}$.

\section{REFERENCES}

Bowman, K. P., and M. D. Fowler, 2015: The diurnal cycle of precipitation in tropical cyclones. J. Climate, 28, 5325-5334, https://doi.org/10.1175/JCLI-D-14-00804.1.

Browner, S. P., W. L. Woodley, and C. G. Griffith, 1977: Diurnal oscillation of the area of cloudiness associated with tropical storms. Mon. Wea. Rev., 105, 856-864, https://doi.org/10.1175/ 1520-0493(1977)105<0856:DOOTAO > 2.0.CO;2.

Bryan, G. H., and J. M. Fritsch, 2002: A benchmark simulation for moist nonhydrostatic numerical models. Mon. Wea. Rev., 130, 2917-2928, https://doi.org/10.1175/1520-0493(2002)130<2917: ABSFMN $>2.0 . \mathrm{CO} ; 2$.

Cronin, T. W., K. A. Emanuel, and P. Molnar, 2015: Island precipitation enhancement and the diurnal cycle in radiativeconvective equilibrium. Quart. J. Roy. Meteor. Soc., 141, 1017-1034, https://doi.org/10.1002/qj.2443.

Dunion, J. P., C. D. Thorncroft, and C. S. Velden, 2014: The tropical cyclone diurnal cycle of mature hurricanes. Mon. Wea. Rev., 142, 3900-3919, https://doi.org/10.1175/ MWR-D-13-00191.1.

,-- , and D. S. Nolan, 2019: Tropical cyclone diurnal cycle signals in a hurricane nature run. Mon. Wea. Rev., 147, 363388, https://doi.org/10.1175/MWR-D-18-0130.1.
Emanuel, K. A., 1986: An air-sea interaction theory for tropical cyclones. Part I: Steady-state maintenance. J. Atmos. Sci., 43, 585-605, https://doi.org/10.1175/1520-0469(1986)043<0585: AASITF $>2.0 . C O ; 2$.

Gray, W. M., and R. W. Jacobson, 1977: Diurnal variation of deep cumulus convection. Mon. Wea. Rev., 105, 11711188, https://doi.org/10.1175/1520-0493(1977)105<1171: DVODCC $>2.0 . \mathrm{CO} ; 2$.

Hodyss, D., and D. S. Nolan, 2007: Linear anelastic equations for atmospheric vortices. J. Atmos. Sci., 64, 2947-2959, https:// doi.org/10.1175/JAS3991.1.

Jordan, C. L., 1958: Mean soundings for the West Indies area. J. Meteor., 15, 91-95, https://doi.org/10.1175/1520-0469(1958) 015<0091:MSFTWI>2.0.CO;2.

Khairoutdinov, M. F., and D. A. Randall, 2003: Cloud resolving modeling of the ARM summer 1997 IOP: Model formulation, results, uncertainties, and sensitivities. J. Atmos. Sci., 60, 607-625, https://doi.org/10.1175/15200469(2003)060<0607:CRMOTA > 2.0.CO;2.

Kossin, J. P., 2002: Daily hurricane variability inferred from GOES infrared imagery. Mon. Wea. Rev., 130, 2260-2270, https://doi.org/ 10.1175/1520-0493(2002)130<2260:DHVIFG>2.0.CO;2.

Mecikalski, J. R., and G. J. Tripoli, 1998: Inertial available kinetic energy and the dynamics of tropical plume formation. Mon. Wea. Rev., 126, 2200-2216, https://doi.org/10.1175/1520-0493(1998) 126<2200:IAKEAT $>2.0 . \mathrm{CO} ; 2$.

Melhauser, C., and F. Zhang, 2014: Diurnal radiation cycle impact on the pregenesis environment of Hurricane Karl (2010). J. Atmos. Sci., 71, 1241-1259, https://doi.org/10.1175/ JAS-D-13-0116.1.

Moon, Y., and D. S. Nolan, 2010: The dynamic response of the hurricane wind field to spiral rainband heating. J. Atmos. Sci., 67, 1779-1805, https://doi.org/10.1175/2010JAS3171.1.

Muramatsu, T., 1983: Diurnal variations of satellite-measured TBB areal distribution and eye diameter of mature typhoons. J. Meteor. Soc. Japan, 61, 77-90, https://doi.org/ 10.2151/jmsj1965.61.1_77.

Navarro, E. L., and G. J. Hakim, 2016: Idealized numerical modeling of the diurnal cycle of tropical cyclones. J. Atmos. Sci., 73, 4189-4201, https://doi.org/10.1175/JAS-D-15-0349.1.

-,- , and H. E. Willoughby, 2017: Balanced response of an axisymmetric tropical cyclone to periodic diurnal heating. J. Atmos. Sci., 74, 3325-3337, https://doi.org/10.1175/ JAS-D-16-0279.1.

Nicholls, M. E., 2015: An investigation of how radiation may cause accelerated rates of tropical cyclogenesis and diurnal cycles 
of convective activity. Atmos. Chem. Phys., 15, 9003-9029, https://doi.org/10.5194/acp-15-9003-2015.

Nolan, D. S., and M. T. Montgomery, 2002: Nonhydrostatic, threedimensional perturbations to balanced, hurricane-like vortices. Part I: Linearized formulation, stability, and evolution. J. Atmos. Sci., 59, 2989-3020, https://doi.org/10.1175/15200469(2002)059<2989:NTDPTB > 2.0.CO;2.

_- and L. D. Grasso, 2003: Nonhydrostatic, three-dimensional perturbations to balanced, hurricane-like vortices. Part II: Symmetric response and nonlinear simulations. J. Atmos. Sci., 60, 2717-2745, https://doi.org/10.1175/1520-0469(2003) $060<2717$ :NTPTBH $>2.0 . \mathrm{CO} ; 2$.

—, Y. Moon, and D. P. Stern, 2007: Tropical cyclone intensification from asymmetric convection: Energetics and ef ficiency. J. Atmos. Sci., 64, 3377-3405, https://doi.org/10.1175/ JAS3988.1.

, R. Atlas, K. T. Bhatia, and L. R. Bucci, 2013: Development and validation of a hurricane nature run using the joint OSSE nature run and the WRF Model. J. Adv. Model. Earth Syst., 5, 382-405, https://doi.org/10.1002/jame.20031.

O'Neill, M. E., D. Perez-Betancourt, and A. A. Wing, 2017: Accessible environments for diurnal-period waves in simulated tropical cyclones. J. Atmos. Sci., 74, 2489-2502, https://doi.org/ 10.1175/JAS-D-16-0294.1.
Randall, D. A., Harshvardhan, and D. A. Dazlich, 1991: Diurnal variability of the hydrologic cycle in a general circulation model. J. Atmos. Sci., 48, 40-62, https://doi.org/10.1175/15200469(1991)048<0040:DVOTHC $>2.0$. CO 2.

Ruppert, J. H., and C. Hohenegger, 2018: Diurnal circulation adjustment and organized deep convection. J. Climate, 31, 48994916, https://doi.org/10.1175/JCLI-D-17-0693.1.

, and M. E. O'Neill, 2019: Diurnal cloud and circulation changes in simulated tropical cyclones. Geophys. Res. Lett., 46, 502-511, https://doi.org/10.1029/2018GL081302.

Tang, X., and F. Zhang, 2016: Impacts of the diurnal radiation cycle on the formation, intensity, and structure of Hurricane Edouard (2014). J. Atmos. Sci., 73, 2871-2892, https://doi.org/ 10.1175/JAS-D-15-0283.1.

— Z. Z.-M. Tan, J. Fang, Y. Q. Sun, and F. Zhang, 2017: Impact of the diurnal radiation cycle on secondary eyewall formation. J. Atmos. Sci., 74, 3079-3098, https://doi.org/10.1175/JAS-D-17-0020.1.

,,--- E. B. Munsell, and F. Zhang, 2019: Impact of the diurnal radiation contrast on the contraction of radius of maximum wind during intensification of Hurricane Edouard (2014). J. Atmos. Sci., 76, 421-432, https://doi.org/10.1175/JAS-D-18-0131.1.

Willoughby, H. E., 2009: Diabatically induced secondary flows in tropical cyclones. Part II: Periodic forcing. Mon. Wea. Rev., 137, 822-835, https://doi.org/10.1175/2008MWR2658.1. 